\title{
A Study of Rehabilitation of CKD Patient's on Hemodialysis in Tribal Population
}

\author{
Dr. Dolly Ajwani Ratre ${ }^{1}$, Rashmi Nande ${ }^{2}$, Navin Kumar Ratre ${ }^{3}$ \\ ${ }^{1}$ BPT, M. Sc. Renal Science and Dialysis Technology, \\ 2B.Sc. Nursing, M. Sc. Renal Science and Dialysis Technology, \\ 3BPT \\ Pt. Jawaharlal Neharu Memorial Medical College \& \\ Dr. Bhimrao Ambedkar Memorial Hospital, Raipur, Chhattisgarh, India
}

\section{INTRODUCTION}

Chronic kidney disease (CKD) worldwide is rising markedly becoming a priority public health problem. The progression of CKD cause functional limitation and severe disability with poor quality of life. The aim of present review was to highlight the effect of rehabilitation in CKD and ESRD subjects. The rehabilitative process is unique in treating disabled people according to a holistic approach with the aim of supporting a person's independent living and autonomy. CKD are associated with an increased risk of functional impairment, independent of age, gender, and comorbidities. Clinicians should counsel patients with CKD including frail elder people to increase physical activity levels and target that regular physical activity including aerobic or endurance exercises training benefits health. In old subjects with CKD and multiple functional impairments, the traditional disease based model should be changed to individualized patient-centered approach that prioritizes patient preferences. Patients receiving haemodialysis have a considerably lower exercise tolerance, functional capacity, and more muscle wasting than healthy subjects or patients with less severe CKD. Exercise training or comprehensive multi-dimensional strategy and goal-oriented intervention should be also provided in ESRD older subjects. Structured prevention programs based on reducing the risk factors for CKD and rehabilitative strategies could reduce disability occurrence. [1](Rehabilitation Medicine and NeuroRehabilitation Unit, Scientific Institute, Hospital 'Casa Sollievodella Sofferenza', San Giovanni Rotondo (Foggia), Italy)

Patients with CKD have a high prevalence of cardiovascular disease associated with or exacerbated by inactivity. This randomized, controlled study investigated whether a renal rehabilitation exercise program for patients with stages 3 or 4 CKD would improve their physical function and quality of life. [2] (clinical journal of the American society of nephrology ).

Exercise rehabilitation has been extensively utilized for patients with severe cardiac, pulmonary, and neurologic diseases. Patients with chronic kidney disease (CKD) as well as those with a functional renal allograft also have physiologic abnormalities that require physical rehabilitation for optimal health. This topic will discuss the underlying causes and treatment of deconditioning in patients with CKD and renal transplant recipients.
Though dialysis maintains life, patients face life-long physical, psychological and social problems related to their illness and treatment. Dialysis can only replace part, but not all, of the renal functions. It cannot correct the co-morbid diseases and it itself incurs its own complications. Anxiety, depression, fear, emotional fluctuation and various psychological stresses are common amongst ESRD patients especially at the early phase of treatment. Dialysis treatment also causes a significant change in daily living, disruption in work schedule and shift in social role which in turn imposes financial, housing, marital and employment problems.

Psychological adaptation and social adjustment are important challenges to renal patients on dialysis therapy.

Rehabilitation has been defined by the World Health Organization (WHO) as "the use of all means aimed at reducing the impact of disabling and handicapping conditions and at enabling people with disabilities to achieve optimal social integration". This definition incorporates clinical rehabilitation but also, importantly, endorses the concept of social participation as requiring a matching of the social environment to the needs of people with disabilities. The overall aim of rehabilitation is to enable people with disabilities to lead the life that they would wish, given any restriction imposed on their activities by impairments resulting from illness or injury as well as from their personal context. The rehabilitative process is unique in treating people according to a holistic approach or a bio-psycho-social model with the aim of supporting a person's independent living and autonomy. Rehabilitation has been demonstrated effective and efficient in reducing the burden of disability and enhancing the opportunities for disabled people. On the base of clinical, affective and functional evaluation, physiatrists have to plan the rehabilitative project including all structural body and functional areas that need of reparative and rehabilitative interventions. Rehabilitative project has to forecast time and intervention type, to individuate recoverable limitations and the functional objectives that can be reached. Furthermore, suitable rehabilitative methods and techniques have to be described in reaching the results as well as the specific role of involved personnel staff in the rehabilitative process. The role of rehabilitation intervention and how much specific strategies can affect functional recovery have to be communicated and discussed with patients and his/her family. Indeed, discussion regarding goals of care and advance care 
planning ought to be common place in executing rehabilitative project. Patients and caregivers should be discouraged from setting unrealistic rehabilitation goals, whenever required functional abilities are not recoverable. The rehabilitation project should be tailored on the individual effective needs of CKD people according to the holistic approach considering stage of disease, complications and co-morbidities. As a patient-centered process, it has to be appropriate to optimize both activity and participation to ameliorate person's quality of life. People with CKD could complain of complex functional impairments and multi-system clinical disorders needing of specialized care and specific rehabilitative interventions. In this case, the rehabilitative project can be only executed by an interdisciplinary approach with a multi-professional team work that include physiatrist, geriatrician, nephrologist and other medical practitioner such as nurses, social worker, and occupational or physical therapist in order to obtain the best benefit by the one's own expert and competent contribute.

\section{Life Options Definition of Renal Rehabilitation}

$>$ Encouragement: Surrounding the patient with a positive attitude.

$>$ Education: Learning about kidney disease and dialysis, and about opportunities and interests.

$>$ Exercise: Essential to recover and maintain physical capacity; improves cardiovascular health.

$>$ Employment: Maintain or return quickly where possible; understand barriers and benefits.

$>$ Evaluation: Repeated assessment of status and changes; important for factual reinforcement (along with regular assessment of functional status and health-related quality of life).(Dialysis therapy)

Rehabilitation personnel and professionals involved in rehabilitation services may develop intervention (treatment) plans based on a thorough assessment of the person with a disability and his/her environment. The intervention plan should also include ways of overcoming environmental and social barriers. It should be age-specific and consider the person's needs and priorities, as well as the economic and sociocultural context in which he/she lives. The goal of a rehabilitation programme may be one or more of the following:

$>$ self-care - interventions that help the person to carry out daily living activities independently, e.g. eating, dressing, toileting, bathing, taking care of the body, etc.

$>$ mobility - interventions that help the person to move around the home and in the community so that he/she can play, go to school or work, etc.

$>$ functional independence - interventions that help the person to develop desired real-life skills which are required on a day-to-day basis. These may include skills required in the home, e.g. meal preparation, housecleaning, childcare, or leisure skills, e.g. reading, painting, travelling, etc.

The Life Options Advisory Council, a multidisciplinary workgroup addressing rehabilitation issues for renal patients, states that the ideal rehabilitation opportunity for a dialysis patient is "a co-ordinated program of medical treatment, education, counseling and dietary and exercise regimens designed to maximize the vocational potential, functional status and quality of life of dialysis patients." The main goals for rehabilitation include employment for those able to work, including patients over age 65 who wish to work; enhanced fitness to improve physical functioning for all patients; improved understanding of adaptation and the options for living well with dialysis; increased control over the effects of kidney disease and dialysis; and resumption of activities enjoyed prior to dialysis.

The Council has identified five "bridges" to rehabilitation that will lead to these desired outcomes. These five bridges (core principles) are identified as the five Es: education, encouragement, employment, exercise, and evaluation. The specifcs of these bridges are well described in the publication Renal Rehabilitation: Bridging the Barriers. The Council has continued to develop materials related to rehabilitation for use by nephrologists, dialysis providers, health care workers, and patients and families. We focus on the core principle of exercise for reversing the effects of physical deconditioning and for optimizing physical functioning and quality of life in patients on dialysis. [3](Hand book of dialysis therapy)

Poor physical functioning among patients with chronic kidney disease (CKD) is highly prevalent. These patients are at increased risk of cardiovascular disease, and are typically able to participate in only the most sedentary of activities. For several reasons, CKD patients have only about half the exercise capacity of normal sedentary individuals. The subsequent de-conditioning isaggravated by systemic effects such as peripheral muscle, cardiac, nutritional and psychosocial dysfunction. Physical inactivity increases the risk of developing heart disease two-fold. In dialysis patients, a reduced ability to exercise has significance not only on quality of life but also on morbidity and mortality.Renal rehabilitation is a therapeutic process, which entails taking a holistic approach to the welfare of the patient with CKD. It is a comprehensive intervention designed to reduce symptoms, and increase functional status and health-related quality of life in CKD patients. Renal rehabilitation requires the coordinated action of a multidisciplinary team in order to deliver an individualised rehabilitation programme to best effect -incorporating exercise training and self-management education. [4](American Journal of Physical Medicine \& Rehabilitation, 2001. 80: p. 13-18.)

\section{Aims and objectives :-}

AIM: This study sought to determine whether a structured, multidisciplinary renal rehabilitation programme combining exercise, education and self-management advice could impact on exercise capacity and functional ability in patients with CKD. The programme consisted of a mixture of aerobic and strengthening exercises, and an education component.

\section{Objectives:-}

$>$ To monitor the impact of renal rehabilitation program in CKD pts.

$>$ To analysis the total no of pts (male and female) are affected by CKD.

$>$ To analysis the other diseases associated with CKD.

$>$ To analysis the difference between reduce ADL activity during assessment than improvement of ADL activity after rehabilitation program.

$>$ To analysis the improvement in lung compliances after rehabilitation.

$>$ To analysis the improvement in muscle strength before and after rehabilitation program. 


\section{Method and material required:- Study design}

The present study is a cross sectional study, that was conducted from the beginning ofmarch 2016 to the end of September, 2016.

\section{Setting of the study}

The study was carried out at the Nephrology Department of Dr B. R. A.M. HOSPITAL RAIPUR.

\section{Population of the study}

Patients from both genders who had CKD on Hemodialysis patient and registered in the Nephrology Department of the hospital. The total number of cases in the department was 50 patients at the end of the study.

\section{Study sample}

Study sample comprised 50subjects CKD on HD patients.

\section{Data Collection}

Data were collected by both direct and indirect methods.

\section{The direct method}

Laboratory investigations including blood sampling after 1214 hour overnight fasting and the following blood tests were done for patients and controls: Creatinine, urea, albumin, cholesterol, triglycerides, HDL, LDL, , hemoglobin, glucose, . Additionally, spot urine samples were tested for ACR. Glomerular filtration rate was estimated in all participating subjects according to the Schwartz formula (Table 2.1). Height, weight, blood pressure were measured for each participant. Body mass index was also calculated for all subjects.

\section{The indirect method}

Face to face interview questionnaire was administrated for both patients and controls (Appendix 1), The questionnaire main content included:

1. Personal data.

2. History of the disease. (for patients)

3. Physical assessment.

\section{statistical analyses}

Data entry and statistical analyses were performed and Results were expressed as frequency or mean \pm SD.

\section{Parameters of clinical study}

50patients (30 males and 20 females with mean age $75 \pm 17$ years) with CKD and dialyzed 45 long-term HD patients,) were included in this one centre study. 50 patients of CKD patients yet dialyzed, the same group of HD patients as in the first study was used and no any healthy subjects were studied. patients at different stages of CKD were included in a cross-sectional study. Patients were separated into five CKD groups according to their estimated glomerular filtration rate [eGFR; Modification of Diet in Renal Disease (MDRD)], based on Kidney Dialysis Outcomes Quality Initiative (K/DOQI), as follows:

stage $5(\mathrm{n}=45 ;$ eGFR $<15 \mathrm{ml} / \mathrm{min})$, stage4 ( $\mathrm{n}=2$; eGFR $15-30 \mathrm{~mL} / \mathrm{min})$, stage $3(\mathrm{n}=2 ; 30-60 \mathrm{~mL} / \mathrm{min})$. stage1 $(\mathrm{n}=1 ;>90 \mathrm{~mL} / \mathrm{min})$.

$>$ CKD stage 2 were analysed $(\mathrm{n}=0$; GFR $>60 \mathrm{~mL} / \mathrm{min})$..
Blood tests and physiological parameters were obtained for each patient at the time of enrolment but before initiation of RRT. Theaetiologies of CKD were CaCx (2\%), DM (12\%), and multifaceted factors (14\%). 42 patients with CKD stage 5 with glomerular filtration rate (eGFR $<15 \mathrm{ml} / \mathrm{min} / 1.73 \mathrm{~m} 2$ ) at the onset of RRT were included. The aetiology of CKD was vasculits (11\%), chronic glomerulonephritis (23\%) hypertension (46\%) and diabetes (12\%). The same group of HD patients as in the first and second study was used for comparison. Written informed consent and laboratory samples were obtained from all subjects according to ethical guidelines. The study was performed in adherence to the principles of the Declaration of DR. B. R. A. M. HOSPITAL RAIPUR and approved by the Institutional Ethical Committee.

\section{Chemicals and reagents}

Chemicals and reagents used in this study are shown in Table 3.1.

\begin{tabular}{|l|}
\hline Reagent \\
\hline Urea \\
Creatinine \\
CBC \\
Microalbumin (urine) \\
Cholesterol \\
Triglycerides \\
HDL- \\
\hline
\end{tabular}

\section{Description}

A proper and adequate rehabilitation program can reverse $\mathrm{m}$ any disabling conditions or can help patients cope withdefici ts that cannot be reversed by medical care. Rehabilitation ad dresses the patient's physical, psychological, andenvironmen tal needs. It is achieved by restoring the patient's physical fu nctions and/or modifying the patient's physicaland social en vironment. The main types of rehabilitation are physical, occ upational, and speech therapy.

Each rehabilitation program is tailored to the individual pati ent's needs and can include one or more types of therapy. Th epatient's physician usually coordinates the efforts of the reh abilitation team, which can include physical, occupational,sp eech, or other therapists; nurses; engineers; physiatrists (ph ysical medicine); psychologists; orthotists (makesdevices suc $\mathrm{h}$ as braces to straighten out curved or poorly shaped bones) ; prosthetists (a therapist who makes artificiallimbs or proth eses); and vocational counselors. Family members are often actively involved in the patient's rehabilitationprogram.

\section{Material required:-}

- Goniometer.(assessment of ROM).

- Hammer (assessment of reflexes).

- Measuring tap. (for muscle girth)

- Manual muscle testing.(for power assessment)

- Glass coma scale.(for consciousness)

- Barthel index.(for ADLs)

- HRmax

\section{$>$ Manual muscle testing:- Principles of Manual Muscle Testing Overview}

An important component of an orthopedic examination is the assessment of muscle strength. The Guide to Physical Therapist Practice lists both manual muscle testing (MMT) and dynamometry as appropriate measures of muscle strength. 
- Manual muscle testing is a procedure for the evaluation of the function and strength of individual muscles and muscle groups based on the effective performance of a movement in relation to the forces of gravity and manual resistance.

- Dynamometry is a method of strength testing using sophisticated strength measuring devices (e.g., handgrip, hand-held, fixed, and isokinetic dynamometry).

When performing strength testing a particular muscle or muscle group is first isolated, then an external force is applied. Resistance applied at the end of the tested range is termed a 'break test'. Resistance applied throughout the range is termed a 'make test'. The results of the strength testing differ depending on the method used. The isometric hold (break test) shows the muscle to have a higher test grade than the resistance given throughout the range (make test). The handheld devices used in dynamometry can help quantify the "breaking force" necessary to depress a limb held in a specific position by the patient.

Whichever testing method is used, the resistance should be applied and released gradually to give the patient sufficient time to offer resistance. Following the manual muscle test, the muscle tested is said to be "weak" or "strong" based upon the muscle's ability to resist the externally applied force over time. A number of grading systems exist for manual muscle testing (Table 1 ).

Table 1: Comparison of MMT grades [3]

\begin{tabular}{|l|l|l|l|}
\hline $\begin{array}{c}\text { rch } \\
\text { Council[4] }\end{array}$ & $\begin{array}{c}\text { Daniels and } \\
\text { Worthingham [5] }\end{array}$ & $\begin{array}{l}\text { Kendall and } \\
\text { McCreary[6] }\end{array}$ & \multicolumn{1}{c|}{ Explanation } \\
\hline $\mathbf{5}$ & Normal(N) & $\mathbf{1 0 0 \%}$ & Holds test position against maximal resistance \\
\hline $4+$ & Good $+(\mathrm{G}+)$ & & Holds test position against moderate to strong pressure \\
\hline $\mathbf{4}$ & Good(G) & $\mathbf{8 0 \%}$ & Holds test position against moderate resistance \\
\hline $4-$ & Good $-(\mathrm{G}-)$ & & Holds test position against slight to moderate pressure \\
\hline $3+$ & Fair $+(\mathrm{F}+)$ & & Holds test position against slight resistance \\
\hline $\mathbf{3}$ & Fair (F) & $\mathbf{5 0 \%}$ & Holds test position against gravity \\
\hline $3-$ & Fair- (F-) & & Gradual release from test position \\
\hline $2+$ & Poor $+(\mathrm{P}+)$ & & $\begin{array}{l}\text { Moves through partial ROM against gravity OR Moves through } \\
\text { complete ROM gravity eliminated and holds against pressure }\end{array}$ \\
\hline $\mathbf{2}$ & Poor(P) & $\mathbf{2 0 \%}$ & Able to move through full ROM gravity eliminated \\
\hline $2-$ & Poor $-(\mathrm{P}-)$ & & Moves through partial ROM gravity eliminated \\
\hline $\mathbf{1}$ & Trace(T) & $\mathbf{5 \%}$ & $\begin{array}{l}\text { No visible movement; palpable or observable tendon } \\
\text { prominence/flicker contraction }\end{array}$ \\
\hline $\mathbf{0}$ & $\mathbf{0}$ & $\mathbf{0 \%}$ & No palpable or observable muscle contraction \\
\hline
\end{tabular}

1. In the Medical Research Council scale, the grades of 0,1 , and 2 are tested in the gravity-minimized position (contraction is perpendicular to the gravitational force). All other grades are tested in the anti-gravity position. The Daniels and Worthingham grading system is considered the more functional of the three grading systems outlined in Table 1 because it tests a motion that utilizes all of the agonists and synergists involved in the motion. The Kendall and McCreary approach is designed to test a specific muscle rather than the motion, and requires both selective recruitment of a muscle by the patient and a sound knowledge of anatomy and kinesiology on the part of the clinician to determine the correct alignment of the muscle fibers.[3] Choosing a particular grading system is based on skill level of the clinician while ensuring consistency for each patient, so that coworkers who may be re-examining the patient are using the same testing method.[5] ( Bohannon, R.W., Measuring knee extensor muscle strength. American Journal of Physical Medicine \& Rehabilitation, 2001. 80: p. 13-18.)

\section{$>$ Barthel Index:}

Barthel index specifically measures the degree of assistance required by an individual on 10 items of mobility and self care ADL. Barthel index is used to monitor functional changes in individual receiving renal rehabilitation in terms of $3 \mathrm{E}$ ie Exercise, Education, Encouragement in predicting the functional outcome as complete independent or complete dependent or require assistance.

\begin{tabular}{|l|l|}
\hline 1 & $\begin{array}{l}\text { FEEDING } \\
10=\text { Independent. Able to apply any necessary device. Feeds in reasonable time. } \\
5=\text { Needs helps (Eg. For cutting) }\end{array}$ \\
\hline 2 & $\begin{array}{l}\text { BATHING } \\
\text { 5=independent }\end{array}$ \\
\hline 3 & $\begin{array}{l}\text { PERSONAL TOILET } \\
5=\text { Independently washes face, comb hair, brushes teeth, saves }\end{array}$ \\
\hline 4 & $\begin{array}{l}\text { DRESSING } \\
\text { 10= independent. Ties shoes, fastens fasteners, applies braces. } \\
5=\text { Needs help, but does at least half of work in reasonable time. }\end{array}$ \\
\hline 5 & $\begin{array}{l}\text { BOWELS } \\
\text { 10= No Accident. Able to use enema or suppository if needed. } \\
\\
5=\text { Occasional accidents or needs help with enema or suppository. }\end{array}$ \\
\hline 6 & $\begin{array}{l}\text { BLADDER } \\
\text { 10= No Accident. Able to care for collecting device if used. }\end{array}$ \\
& $5=$ Occasional accidents or needs help with device. \\
\hline
\end{tabular}


International Journal of Trend in Scientific Research and Development (IJTSRD) @ www.ijtsrd.com eISSN: 2456-6470

\begin{tabular}{|c|c|}
\hline 7 & $\begin{array}{l}\text { TOILET TRANSFERS } \\
\text { 10= Independent with toilet or bedpan. Handles clothes, wipes, flushes, or cleans pan. } \\
5=\text { Needs help for balance, handling cloths or toilet paper. }\end{array}$ \\
\hline 8 & $\begin{array}{l}\text { TRANSFERS-CHAIR \& BED } \\
\text { 15= Independent, including locking of wheel chair, lifting footrests. } \\
\text { 10= Minimum assistance or supervision. } \\
5=\text { Able to sit, but needs maximum assistance to transfer. }\end{array}$ \\
\hline 9 & $\begin{array}{l}\text { AMBULATION } \\
\text { 15= Independent for } 50 \text { Yards. May use assistive device except for rolling walking. } \\
\text { 10= With help, } 50 \text { yards. } \\
5=\text { Independent with wheelchair for } 50 \text { yards if unable to walk. }\end{array}$ \\
\hline 10 & $\begin{array}{l}\text { STAIR CLIMBING } \\
\text { 10= Independent. May use assistive devices. } \\
\text { 5= Needs help or supervision. }\end{array}$ \\
\hline & 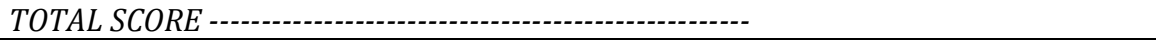 \\
\hline
\end{tabular}

- A score of zero (0) is given in any category in which the patient does not achieve the stated criterion. [6] ( Physical Rehabilitation, $5^{\text {th }}$ edition, Susan B O'Sullivan/ Mahoney, F, and Barthel, D, PP 62-65)

\section{Modalities:-}

used fused for treatment to reducing pain and other complication of CKD.

$>$ Ultrasound.(reduce inflammation and pain)

$>$ TENS.(improving sensory and motor nerve impulses)

$>$ IFT. (re-educate muscle action)

\section{Exercise equipment:-}

$>$ Weight cuff/doumbles

$>$ Spirometer.

$>$ Pulley.

$>$ Shoulder wheel.

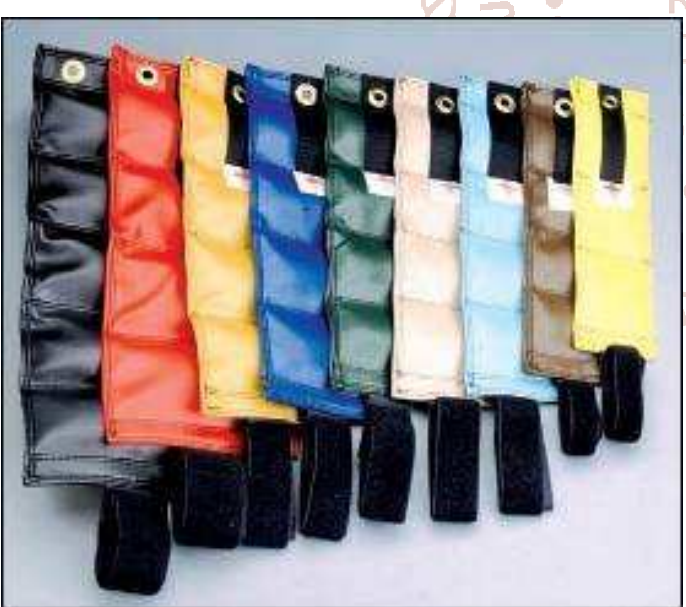

figure1:- different wt. cuffes using for muscle strength.

$>$ Spirometer:-

How to Use an incentive Spirometer

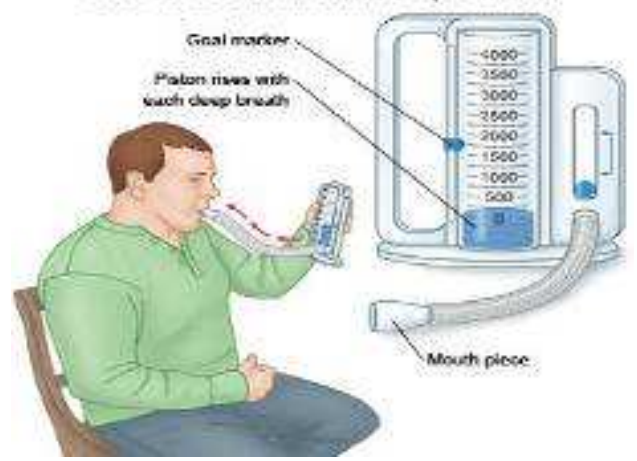

Figure 2:- shows lung compliance exercice
Pulley:-

exercise guide

Some of the exercises that can be performanced at this machine are.
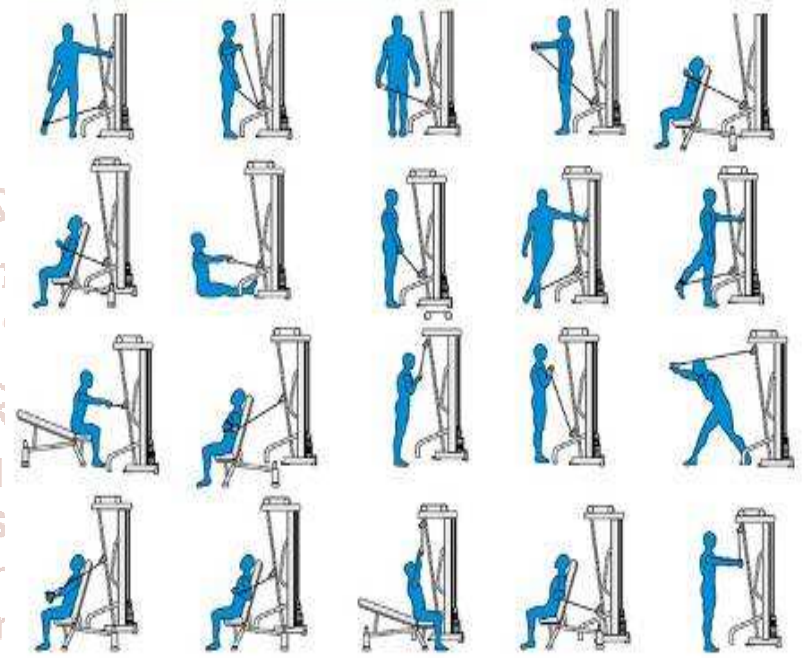

Figure 3:- shows different pattern of pulley exersices

Figure 4:- Shoulder Wheel

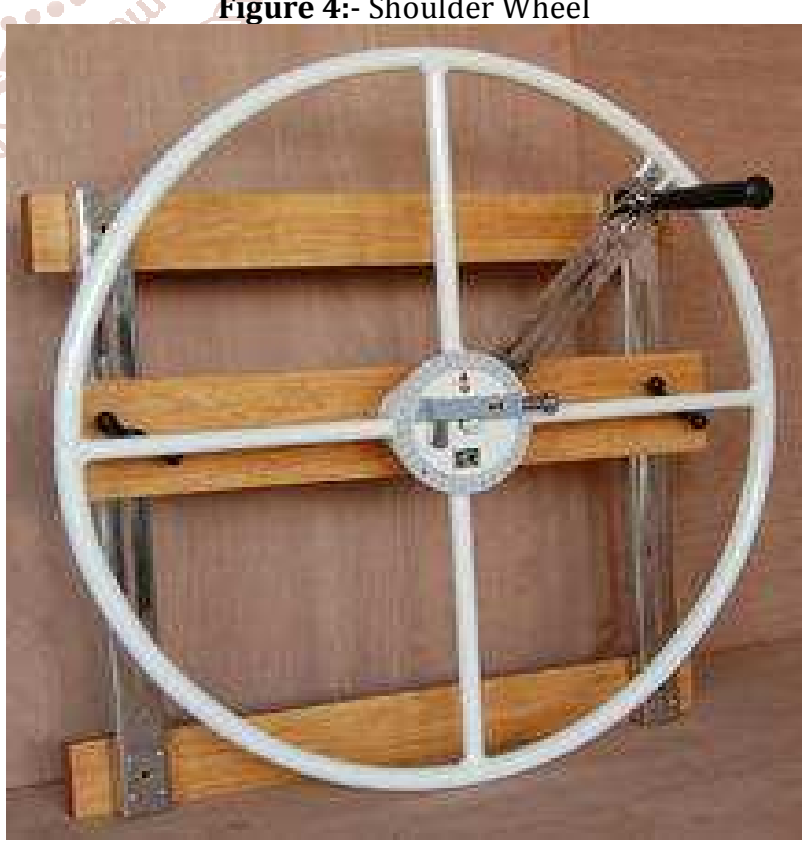

A $100 \mathrm{~cm}$ diameter, tubular steel constructed Wheel is fitted with Resistance mechanism and 360 degree scale. The wheel revolves smoothly with C.I. drum for shoulder exercises. 
Figure 5:- Shoulder Exerciser, Combo unit

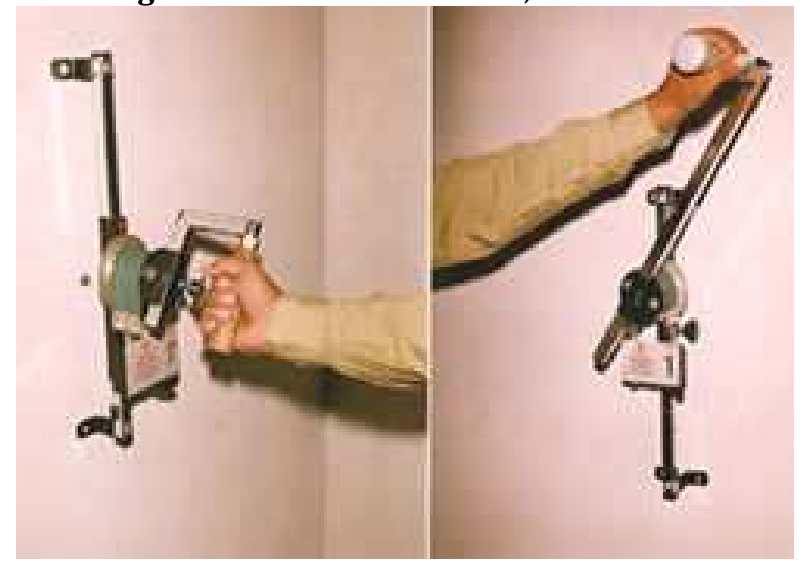

A combo unit for Shoulder \& Supination-Pronation exercises is fitted with Resistance Scale, Circumference Scale \& a 360 deg movement scale to measure \& record the improvement. Unit is suitable to improve circulation, muscle strength, joint range of motion and coordination. Used in the same way as a conventional shoulder wheel and rotary wrist machine.

\section{Physical Functioning}

Physical functioning is often used as a term to encompass many concepts. Physical functioning is best defined as an individual's ability to perform activities required in their daily lives. Physical functioning is determined by many factors, including physical fitness (cardio respiratory fitness, strength, and flexibility), sensory function, clinical condition, environmental factors, and behavioral factors. Physical fitness is a set of attributes people have or achieve that relates to the ability to perform physical activity. One of these attributes is cardio respiratory fitness (often referred to As exercise capacity), which relates to the ability of the cardiac, circulatory, and respiratory systems to supply and use oxygen during sustained physical activity.

Physical functioning can be improved with regular physical activity or exercise training. Exercise (physical activity) is defined as bodily movement produced by the contraction of skeletal muscle that substantially increases energy expenditure.

\section{Definition of Terms Related to Physical Functioning}

$>$ Physical functioning: An individual's ability to perform activities required in daily life.

$>$ Physical fitness: A set of attributes that relates to the ability to perform physical activity (e.g., cardio respiratory fitness, muscle strength, flexibility).

$>$ Exercise capacity cardio respiratory fitness): Attribute that relates to the ability of the cardiac, circulatory, and respiratory systems to supply and use oxygen during sustained physical activity.

$>$ Exercise (physical activity): Bodily movement produced by muscle movement that substantially increases energy expenditure

$>$ Exercise training: Planned, structured, and repetitive bodily movement done to improve physical fitness or to obtain other health benefits.

Exercise training is planned, structured, and repetitive bodily movement done to improve or maintain one or more components of physical fitness or to obtain other health benefits. Increased physicalactivity can be considered exercise training, although increased physical activity can also result from unstructured increases in movements throughout the day. The use of the term physical activity may be less intimidating to dialysis patients who are typically elderly and often frail or chronically fatigued. However, exercise training is appropriate and recommended for dialysis patients due to their extremely low levels of physical functioning and exercise capacity.

\section{Assessment of Physical Functioning}

Given the multiple determinants of physical functioning (i.e., cardio respiratory fitness, strength, sensory function, clinical status, and environmental and behavioral factors), no one measure can cover all areas. Assessment of functioning can range from objective laboratory measures of cardio respiratory fitness to questionnaires of self-reported physical functioning that include questions relating to the ability to perform activities that range from basic self-care to household activities and more strenuous tasks.

cardio respiratory fitness (exercise capacity) is objectively measured using laboratory estimates or actual measures of oxygen uptake during a maximal exercise test performed on a cycle ergometer or treadmill (V02max). There are objective criteria for a chievement of V02max, many of which are not achieved by patients with chronic disease (who are often limited by symptoms such as skeletal muscle weakness or shortness of breath). In such cases, the measure of exercise capacity should be referred to as V02peak, or symptom-limited V02peak. Because of the expertise and technical requirements of exercise testing, it may not be practical for routine clinical evaluation of physical functioning.

Thus, other measures are available for assessment. The growing interest in physical functioning in older and diseased populations has led to development of tests that measure physical performance of standardized tasks such as walking (6-minute walk, gait speed), balancing, reaching, rising from a chair, and climbing stairs. These tests are more realistic for the majority of patients, are less expensive, do not require specialized equipment or testing personnel, and are easily performed in a clinical setting. These tests are referred to as physical performance tests. They are not direct measures of cardio respiratory fitness, strength, or flexibility but are indicators of these physical fitness measures. Selfreported physical functioning can also be evaluated using questionnaires, such as the SF-36 Health Status questionnaire-which assess level of difficulty performing activities of daily living, instrumental activities of daily living, and more strenuous activities.[10] (hand book of dialysis therapy)

\section{Review of literature:-}

In 1993, with support from Amgen, the Life Options program was started-and the Life Options Rehabilitation Advisory Council (LORAC), composed of experts in dialysis care and psychosocial research, was established to lead it. The Council's members set out to defi ne an orderly structure for rehabilitation efforts that would include physical, psychosocial, and vocationa rehabilitation arenas-and that would provide guidance to clinicians and facilities undertaking it. This program is not the only such effort, but the organization of ideas and the subsequently reported successful programs make it an easy model for application to most ESRD treatment sites-as well as to other chronic disease settings.[11](hand book of dialysis therapy) 
Greenwood, S, Lindup, H, Taylor, K, Garrod, R, Macdougall, IKing's College Hospital, London shows: Exercise testing results and functional ability scores for the 64 patients who had complete data pre and post rehabilitation are as follows: ISWT improved by 54\% $(\mathrm{p}<0.001)$, STS60 by $40 \% \quad(\mathrm{p}<0.001)$, TUAG by $28 \%$ $(\mathrm{p}<0.001)$, SCD by $33 \%(\mathrm{p}<0.001)$, RPEHDT by $42 \%$ $(\mathrm{p}<0.001)$, and DASI by $67 \%(\mathrm{p}<0.001)$ following 12 weeks of rehabilitation.

Exercise programmes have been combined successfully with other therapeutic measures. In one study, 79 HD patients followed a programme of resistance training with or without anabolic steroids. The steroids signifycantly increased lean body mass with an additive effect on top of that of resistance exercise alone . In another study Pupim et al. demonstrated that intradialytic parenteral nutrition combined with aerobic exercise increased protein synthesis and reduced the rate of proteolysis significantly more than intradialytic parenteral nutrition alone.

STUDY OF BARTHEL SCORE AMONG CKD PATIENTS BELONGING FROM TRIBAL AREAS IN TERTIARY CARE HOSPITAL, CHHATTISGARH, this study shows of patients with Chronic Kidney Disease were having altered functional status. In this study, male CKD patients had better mean Barthel Index score than females. CKD patients those who were having diabetes have low BI. Patients not on dialysis have better Barthel Index score than patients on maintenance hemodialysis.

A 12-week/24-session renal rehabilitation exercise program improved physical capacity and quality of life in patients with CKD stages 3 and 4 . Longer follow-up is needed to determine if these findings will translate into decreased mortality rates.[12] ( Dr. James C. Wasserman, Division of Nephrology and Transplantation, Maine Medical Center, 22 Bramhall Street, Portland, ME 04102-3175)

Because MCS scores have been shown in other studies to be inversely related to morbidity and mortality, this finding suggests that the introduction of rehabilitation interventions into the dialysis care regimen may prove beneficial.[13](Copyright 2002 by the National Kidney Foundation, IRenal rehabilitation and improved patient outcomes in Texas dialysis facilities. Curtin RB ${ }^{1}$, Klag MJ, Bultman DC, Schatell D.)

In another study by Painter et al., in which hematocrit was increased using r-HuEPO from $33 \%$ to 40 to $42 \%$, there was no change in VO2peak unless the patients were involved in exercise training. In fact, even with this near-normalization of hematocrit plus exercise training, V02peak remained surprisingly low compared to age-predicted values (average $56.8 \pm 20.6 \%$ of age- and gender-predicted levels).[14] (hand bookof dialysis therapy)

Cycling exercise before or during dialysis is an option in some treatment centers. Exercise training during dialysis treatments may improve solute removal by increasing blood flow to muscle and efflux of urea and other toxins into the vascular compartment where they can be removed (Parsons et al. 2006). At the same time, there is a possibility of reduced exercise tolerance during dialysis resulting from fluid and electrolyte shifts, and exercise could exacerbate dialysis-associated hypotension (Johansen 2007). It appears; however, that exercise is generally well tolerated within the first 1-2 hours of a hemodialysis (HD) session (Painter et al. 2002). [15](International encyclopedia of rehabilitation)

Kouidi et al. enrolled seven patients who were receiving long-term hemodialysis into a 6-mo exercise rehabilitation program that included aerobic exercise and strengthening exercise. The program consisted of 90-min sessions three times per week on nondialysis days. Specifically, the training routine included a 10-min warm-up followed by $50 \mathrm{~min}$ of aerobic exercises, $10 \mathrm{~min}$ of low-weight resistance exercise, $10 \mathrm{~min}$ of stretching exercises, and $10 \mathrm{~min}$ of cool-down. They examined the effect of this program on $\mathrm{VO}_{2 \text { peak }}$ and on muscle morphology. The program resulted in an average increase in $\mathrm{VO}_{2 \text { peak }}$ of $48 \%$, an increase that is greater than any program involving a erobic exercise training alone. They also reported a remarkable improvement in muscle atrophy, with a $25.9 \%$ increase in the mean area for type I fibers and a $23.7 \%$ increase in mean area of type II fibers. Although they characterized their training program as "mainly of aerobic type," the notable muscle hypertrophy and the stunning improvement in $\mathrm{VO}_{2 \text { peak }}$ suggest that the strength training portion may have contributed important additive or synergistic effects to the aerobic training. It is possible that muscle atrophy in some patients with ESRD is so severe as to limit $\mathrm{VO}_{2 \text { peak }}$ because of the small mass of working muscle. Unfortunately, the design of this study did not allow the separate contributions of aerobic and resistance training to -be delineated.

The same group of investigators also examined heart rate variability before and after the same exercise training program in 30 exercising patients and 30 sedentary control subjects $(\underline{70})$. They found that heart rate variability increased among the exercisers, suggesting improved autonomic control of the heart and reduced risk for arrhythmia

Two studies were designed specifically to investigate the effects of exercise on BP control in patients who were on hemodialysis. In the first, patients were nonrandomly assigned to a 6-mo cycling exercise program during dialysis ( 40 exercisers and 35 control subjects). At the end of 6 mo, $24(60 \%)$ patients were still participating in the exercise program. The patients who completed 6 mo of training had no changes in BP before or after dialysis but were, on average, taking fewer antihypertensive medications to achieve that BP than before the program, whereas the control group did not have any significant change in BP or antihypertensive medications. The second study also involved cycling exercise during $\mathrm{HD}$ and enrolled 19 patients, 13 of whom completed at least 3 mo of training. Predialysis and interdialytic ambulatory systolic and diastolic BP decreased after 4 mo of training, a finding that persisted after 6 mo of training. The two studies to date that were specifically designed to evaluate BP control demonstrated a beneficial effect of exercise training. [16] (Journal of the American society of Nephrology)

Johansen et al. carried out a controlled study consisting of 12-week modarateinten-sity lower extremity resistance exercise training and found no change with DEXA; how- ever they found that quadriceps muscle area measured by magnetic resonance imaging (MRI) have improved. This change accompanies increases in body weight and fat mass. 
In another controlled study, Cheema et al. found no improvement in computerized tomograpgy (CT) scan in skeleton muscle amount with 12-week high intensity progres-sive resistance training routinely applied during hemodialysis. Similarly, the follow-up after 24 weeks found no additional benefit. Kopple et al.examined the effects of different exercise training (strength, endurance and combination of both) in RNA levels in muscle genes. Although there was no significant difference in non-fat body mass (LBM) at the end of 6 month individual exercises, it was observed that exercise training in hemodialysis patients increased m RNA changes in skeleton muscle and muscle insu-lin-like growth factor-1 (IGF-I) protein. This factor is important as it accelerates protein anabolism. On the other hand, although the effects of resistance exercise on muscle quantity and quality in hemodialysis patients have been shown, strength and physical function cannot be mainPhysiotherapy in the Patients on Hemodialysis tained in these patients. Although the causes are not clear, lack of nutritional support partic-ularly during hemodialysis is held responsible.

Balakrishan et al. analyzed aerobic effects of 12-week high intensity training in random-ized controlled chronic kidney disease stage 3 and 4 patients with low protein diet (approxi-mately $0.6 \mathrm{~g} / \mathrm{kg} / \mathrm{day}$ ) to examine the effect of resistance training on mtDNA copy number and to identify combination with skeleton muscle phenotype (measurement of muscle mass and strength). Resistance Exercise Training Group Participants exercised three times per week under supervision. Each session lasted approxi-mately 45 minutes and included the following: 5 minutes warm-up, 35 minutes resistance training on chest and leg press, M.latissimus pulldown, knee extension, and flexion pneu-matic resistance training machines (Keiser Sports Health Equipment Inc., Fresno, CA), and 5 minutes cool-down. Participants performed three sets of eight repetitions on each machine per session. Training intensity was targeted at $80 \%$ of one repetition maximum (1RM) and progressively increased per participants' self-perceived level of exertion using a Rating of Perceived Exertion Scale. Cool-down included five to eight upper and lower bodystretching and flexibility exercises. Attention-Control Group Participants met and performed the same stretching and flexibility exercises as those used during cool-down in the resistance exercise training group. In conclusion it was found that oxidative metabolic capacity increased in uremic skeleton muscle. There was no relationship between mitochondrial content and insulin resistance. Physiotherapy in the Patients on Hemodialysis The factors affecting anabolism such as energy intake and IGF-1 concentrations in circulation were found to be positively correlated with the changes in mtDNA copy number. Therefore, mechanisms of anabolic and genomic factors on mitochondrial functions should be analyzed and well understood.

\section{Observation}

\subsection{Characteristics of the study population}

Total 50 patients admitted with CKD, were interviewed and assessed for rehablitation. Out of 50 patients, $60 \%$ were male and $40 \%$ were female. Mean age was 43.98 years; the youngest patient was 10 \& the eldest was 85 years old. None of the patients was in CKD stage $2,2 \%$ patient was in stage 1 , $4 \%$ patients were in stage $3,4 \%$ patients were falling in stage 4 and 45 patients were suffering from stage 5 CKD
(Staging of CKD was done according to KDOQI clinical practice guidelines).

Etiology of CKD was diabetes mellitus, obstructive nephropathy, Undetermined 16\%, 10\%, 36\% respectively while lupus nephritis, polycystic kidney disease, sickle cell disease patients was $1(2 \%)$ each.

\subsubsection{Socio-demographic characteristics of the study population}

The distribution of the study population by gender showed that males represented $30(60 \%)$ and of the females represented $20(40 \%)$ of the patients.

\begin{tabular}{|l|c|c|}
\hline S. No. & Stage of CKD & No, of pts. \\
\hline 1. & Stage 1 & $01(2 \%)$ \\
\hline 2. & Stage 2 & $0(0 \%)$ \\
\hline 3. & Stage 3 & $02(4 \%)$ \\
\hline 4. & Stage 4 & $02(4 \%)$ \\
\hline $\mathbf{5 .}$ & Stage 5 & $45(90 \%)$ \\
\hline
\end{tabular}

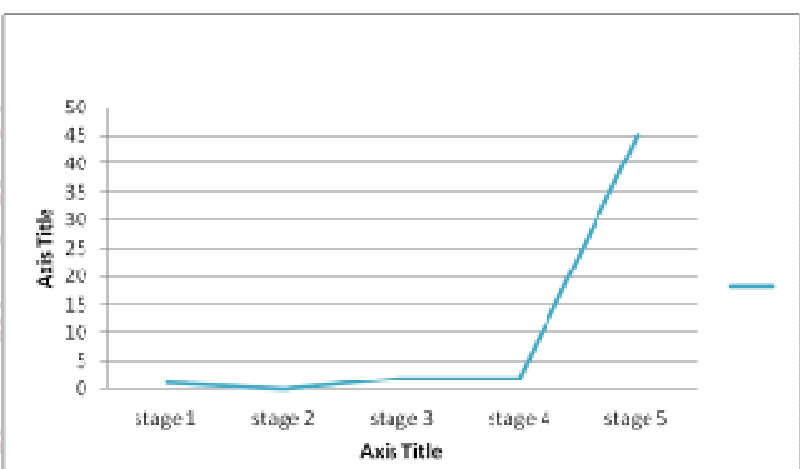

Figure6:- figure shows stage of CKD patients

5.1.2 Male to female ratio among study population:-

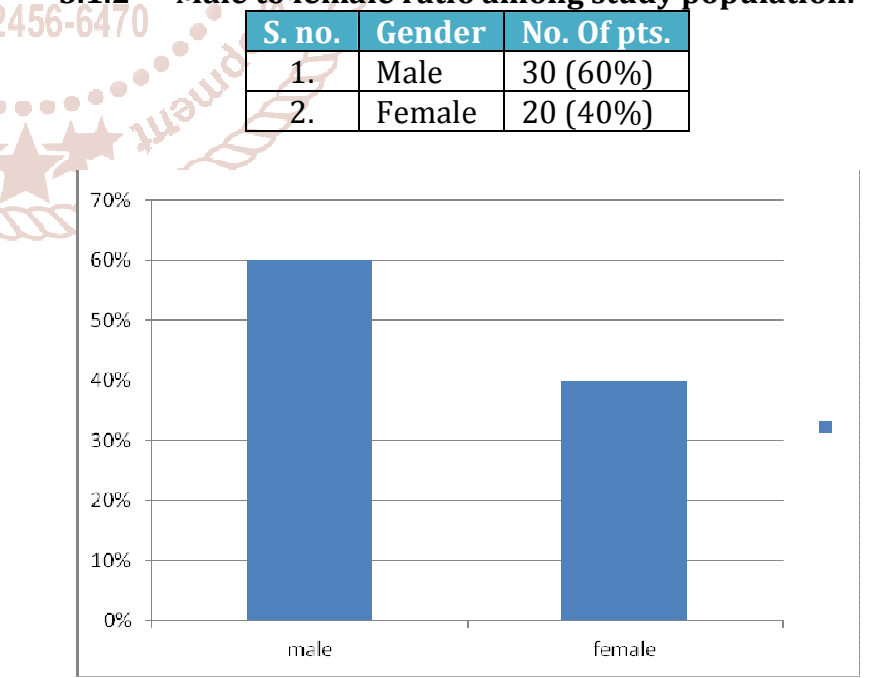

Figure 7:- figure shows male to female ratio

5.1.3 Other different diseases associated with CKD:-

\begin{tabular}{|c|c|c|}
\hline s. no. & Other diseases & $\%$ of total data \\
\hline 1. & CaCx & $3(6 \%)$ \\
\hline 2. & DM & $18(36 \%)$ \\
\hline 3. & RPD & $6(12 \%)$ \\
\hline 4. & GN & $18(36 \%)$ \\
\hline 5. & PTB & $6(12 \%)$ \\
\hline
\end{tabular}




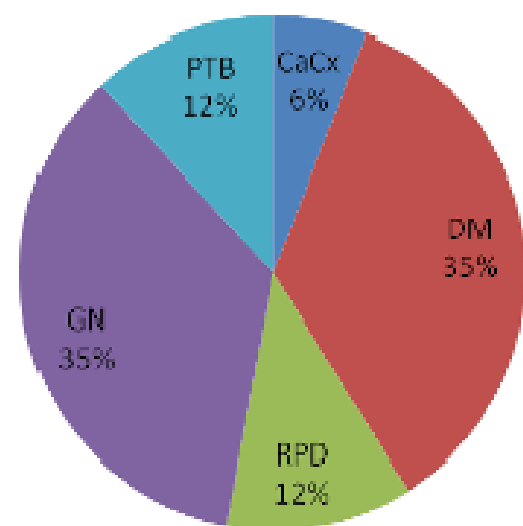

Figure 8:- shows other diseases associated with CKD

5.1.4 Complication related to CKD:-

\begin{tabular}{|c|c|c|c|}
\hline $\begin{array}{c}\text { s. } \\
\text { no. }\end{array}$ & Complication & $\begin{array}{c}\text { No. of } \\
\text { pts. }\end{array}$ & $\begin{array}{c}\% \text { of total } \\
\text { pts. }\end{array}$ \\
\hline 1 & Muscular weakness & 9 & $18 \%$ \\
\hline 2 & DVT & 5 & $10 \%$ \\
\hline 3 & Bed sore & 7 & $14 \%$ \\
\hline 4 & $\begin{array}{l}\text { lung :- } \\
\text { 1.pneumonitis } \\
\text { 2.pleural effusion }\end{array}$ & $\begin{array}{l}6 \\
9 \\
\end{array}$ & $\begin{array}{l}12 \% \\
18 \%\end{array}$ \\
\hline 5 & $\begin{array}{l}\text { Pain:- } \\
\text { 1.backache } \\
\text { 2. knee pain }\end{array}$ & $\begin{array}{l}18 \\
10\end{array}$ & $20 \%$ \\
\hline
\end{tabular}

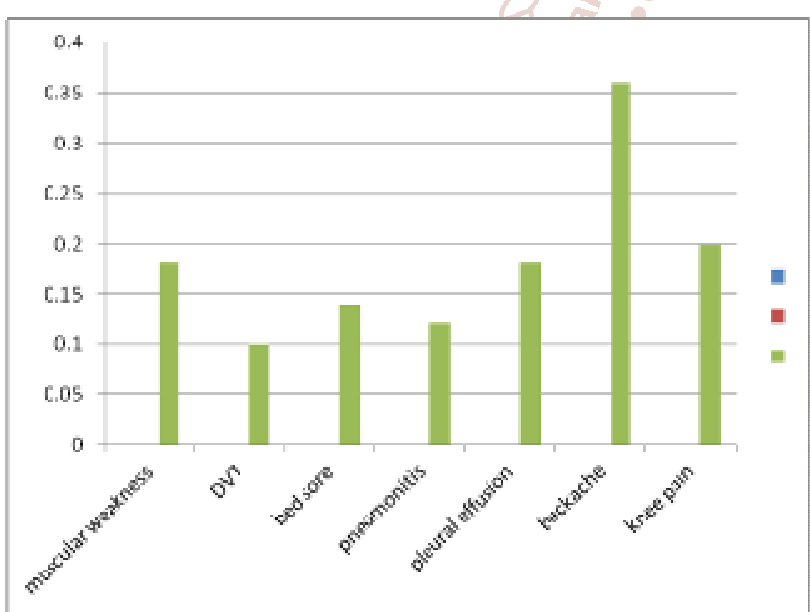

Figure 9;- shows complication of CKD pts no HD

\subsection{Result:-}

Based on the above study results and discussion it can be concluded that majority of patients with Chronic Kidney Disease were having altered functional status. In this study, male CKD patients had better mean of rehablitation than females. CKD patients those who were having diabetes have low result of physiotherapy treatment. Patients not on dialysis have better functional activity than patients on maintenance hem dialysis. 1.During analysis we seen Out of all 50 patients were found that 0 pts seen as 1, 2, 3,10 score , $12 \%$ patients scored $6,16 \%$ patients scored $5,24 \%$ patients scored $6,18 \%$ patients scored 7 and $11 \%$ patients scored 8 and $8 \%$ patient scored 9 .

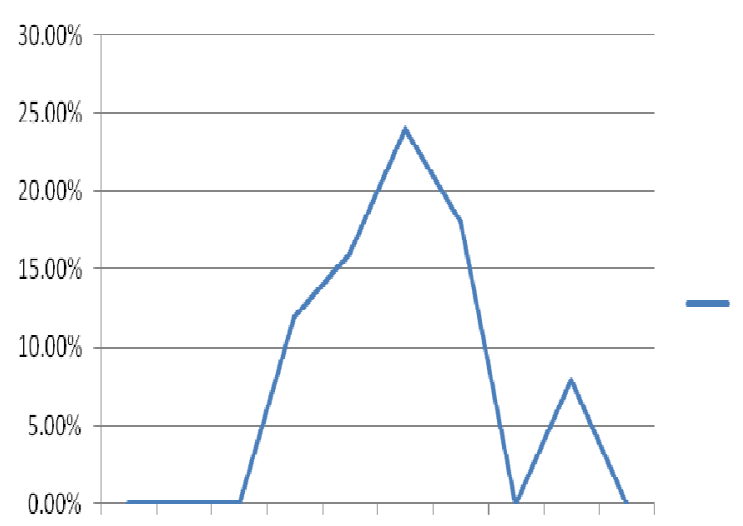

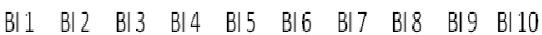

Figure 10:-figure shows BI (ADL) pre rehabilitation of patients with CKD

$>$ After rehabilitation program patients shows improvement and perform their activities, some patients scored as 10 i.e, $12 \%$ patients scored $6,16 \%$ patients scored 7 and $8,8 \%$ patients

$>$ scored 9 and $6 \%$ patient scored 10 which shown in figure.

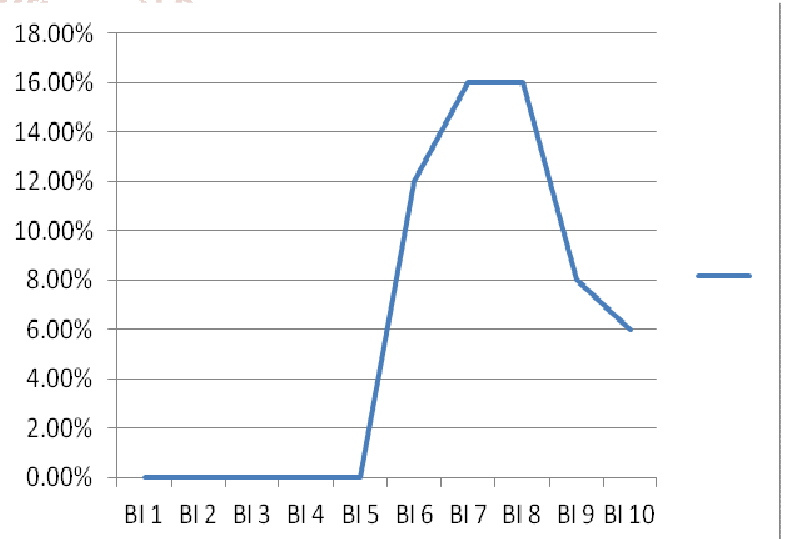

Figure 11:- figure shows recovery state of $\mathrm{BI}$.

1. During analysis of muscle power by MMT $6 \%$ patients graded as fair, $16 \%$ patients graded as average, and 78\% patients graded as good.

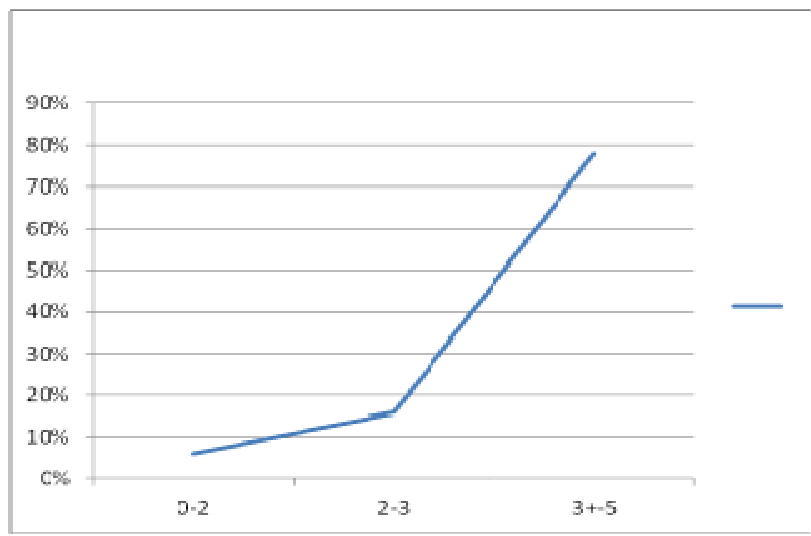

Figure12:- shows muscular weakness to CKD pts

$>$ After rehabilitation muscular strength increases and patient graded as $2 \%$ graded fair, $20 \%$ graded average, $84 \%$ graded good after recovery phase. 
International Journal of Trend in Scientific Research and Development (IJTSRD) @ www.ijtsrd.com eISSN: 2456-6470

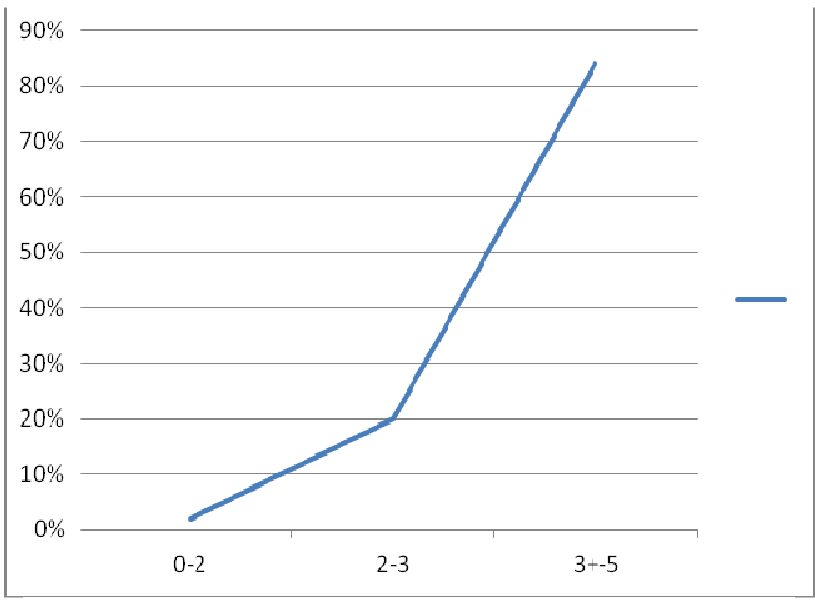

Figure 13:- shows improvement in muscular weakness after rehabilitation.

2. Patient with CKD have pain in different joints but more seen as36\% patient had back pain, $20 \%$ patient had knee pain, and $6 \%$ patient had shoulder pain shown in following graf.

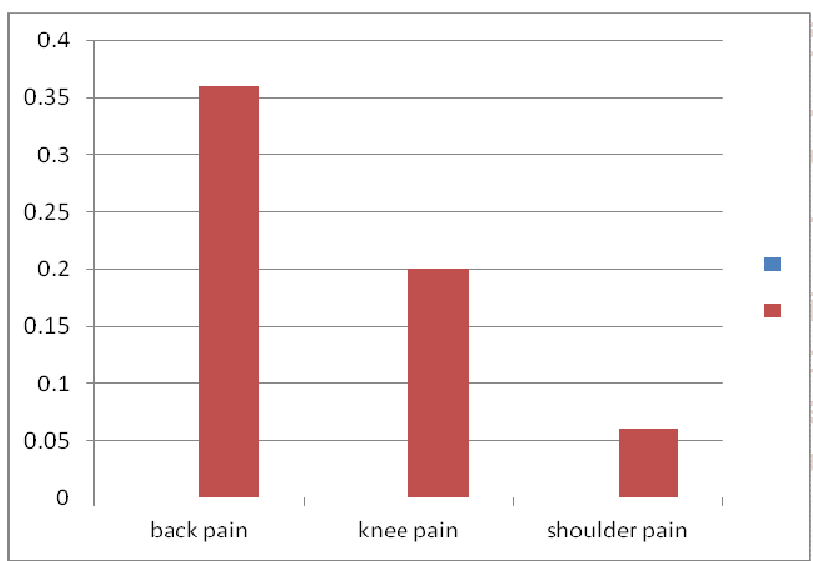

Figure14:- shows pain in different joint before rehabilitation.

$>$ After rehabilitation program which include modalities and exercises that improves in pain as $24 \%$ in back pain, $16 \%$ in knee pain, $4 \%$ in shoulder pain shown in figure

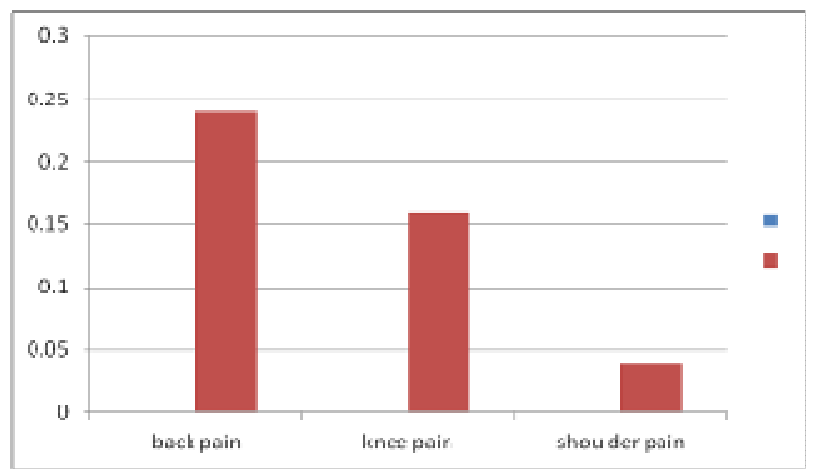

Figure 15:- shows improvement after modality and rehabilitation.

3. Out of 50 patient shows pulmonary complications as $12 \%$ patient had pneumonitis, where as $18 \%$ patient had pleural effusion.

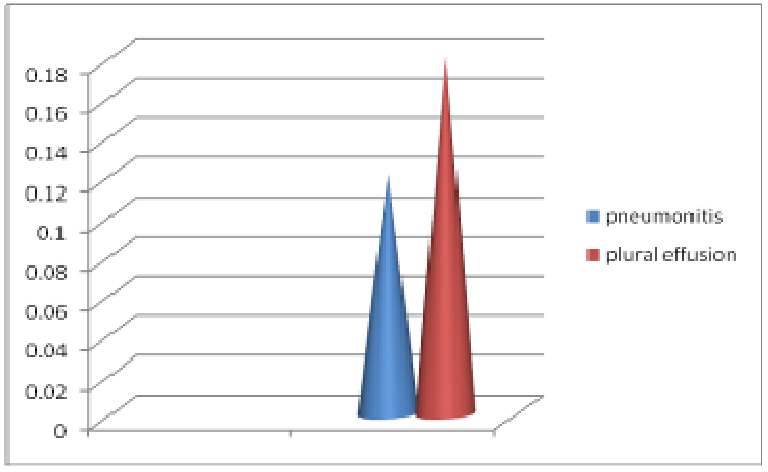

Figure 16:- shows pulmonary complication of CKD patients

After rehabilitation program that includes breathing exercises, different comfortable position, and different position of postural drainage results improvement in lung compliance.

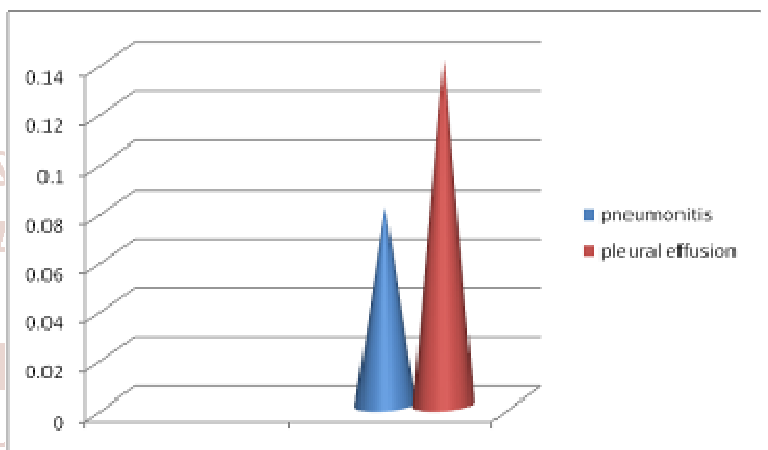

Figure17:- shows improvement in lung compliance after rehabilitation.

6. Discussion :-

The physiological response to exercise is dependent on the intensity, duration and frequency of the exercise as well as the environmental conditions. During physical exercise, requirements for oxygen and substrate in skeletal muscle are increased, as are the removal of metabolites and carbon dioxide. Chemical, mechanical and thermal stimuli affect alterations in metabolic, cardiovascular and ventilatory function in order to meet these increased demands.

Chronic kidney disease (CKD) is strongly associated with cardiovascular disease and muscle wasting, arising from numerous factors associated with declining renal function and lifestyle factors. Exercise has the ability to impact beneficially on the comorbidities associated with CKD and is accepted as an important intervention in the treatment, prevention and rehabilitation of other chronic diseases, however, the role of exercise in CKD is overlooked, with the provision of rehabilitation programmes well behind those of cardiology and respiratory services. Whilst there is now a large evidence base demonstrating the efficacy and safety of exercise training interventions in patients receiving dialysis, and this is now becoming incorporated into clinical guidelines for treatment of dialysis patients, there is a paucity of research evaluating the effectiveness of exercise in patients with CKD who are not on dialysis. Despite this, existing studies indicate that exercise can improve physical functioning and impact positively on the mediators of comorbid diseases and upstream factors associated with progression of renal disease. Although preliminary evidence appears positive, more research is required to identify the 
best modes, frequency and intensities of exercise in order to optimise exercise prescription in pre-dialysis CKD patients. This review summarizes what is known about the main effects of exercise in pre-dialysis CKD patients, discusses the potential of exercise in the rehabilitation and treatment of disease. [17](C) 2014 Asian Pacific Society of Nephrology).

Patients with chronic kidney disease (CKD) are inactive and have reduced physical functioning and performance. Aerobic exercise interventions have been shown to increase maximal oxygen consumption in selected patients. In addition, preliminary evidence, although mixed, suggests that aerobic exercise training can improve blood pressure control, lipid profiles and mental health in this population. A few larger studies are now available showing that aerobic training can also improve physical functioning and performance. The impact on survival or hospitalisation has not been determined. Resistance exercise training, although less studied, appears to increase muscle strength and size and may also improve functioning. There have been several reports of successful combined exercise interventions, but the designs have not allowed evaluation of the relative benefits of aerobic and resistance training on physical functioning. Despite the evidence that exercise is safe and beneficial in patients with CKD, dialysis patients remain inactive, and exercise assessment, counselling and training is not widely offered to patients with CKD. Studies of the barriers to patient participation in exercise and to provider assessment and recommendations are needed so that more widely generalisable interventions can be developed. However, in the interim, patients should be encouraged to participate in moderate physical activity to meet the US Surgeon General's recommendations. Patients who are weak can benefit from strength-training interventions. Resistance and aerobic exercise programmes should be initiated at relatively low intensity in patients with CKD and progressed as slowly as tolerated in order to avoid injury and discontinuation of exercise. For patients on haemodialysis, incorporation of exercise into the dialysis session may increase patient participation and tolerance of exercise.

\section{Effect of exercise in different systems:- Respiratory system}

During exercise, ventilation might increase from resting values of around 5-6 litre min-1 to >100 litre min-1. Ventilation increases linearly with increases in work rate at submaximal exercise intensities. Oxygen consumption also increases linearly with increasing work rate at submaximal intensities. In an average young male, resting oxygen consumption is about $250 \mathrm{ml} \mathrm{min-1}$ and in an endurance athlete oxygen consumption during very high intensity exercise might reach $5000 \mathrm{ml} \mathrm{min-1}$. The increase in pulmonary ventilation is attributable to a combination of increases in tidal volume and respiratory rate and closely matches the increase in oxygen uptake and carbon dioxide output. Breathing capacity, however, does not reach its maximum even during strenuous exercise and it is not responsible for the limitation in oxygen delivery to muscles seen during high intensity activity. Haemoglobin continues to be fully saturated with oxygen throughout exercise in people with normal respiratory function.

\section{Changes in arterial blood gases}

The changes which occur in arterial $\mathrm{pH}, \mathrm{PO}_{2}$ and $\mathrm{PCO}_{2}$ values during exercise are usually small.
Arterial $P_{2} \mathrm{O}_{2}$ often rises slightly because of hyperventilation although it may eventually fall at high work rates. During vigorous exercise, when sufficient oxygen for flux through the Krebs cycle is not available, the increased reliance on glycolysis results in increased accumulation of lactic acid, which initially leads to an increase in $\mathrm{PaCO}_{2}$. However, this is counteracted by the stimulation of ventilation and as a result $\mathrm{PaCO}_{2}$ is decreased. This provides some respiratory compensation for further lactic acid production and prevents a decline in blood $\mathrm{pH}$, which remains nearly constant during moderate exercise.

\section{Changes in ventilation}

Ventilation increases abruptly in the initial stages of exercise and is then followed by a more gradual increase. The rapid rise in ventilation at the onset of exercise is thought to be attributable to motor centre activity and afferent impulses from proprioceptors of the limbs, joints and muscles. The mechanism of stimulation following this first stage is not completely understood. Arterial oxygen and carbon dioxide tensions are not sufficiently abnormal to stimulate respiration during exercise. Suggestions have been made that the sensitivity of peripheral chemoreceptors to oscillations in $\mathrm{PaO}_{2}$ and $\mathrm{PaCO}_{2}$ is responsible for increasing ventilation, even though the absolute values remain stable. Central chemoreceptors may be readjusted to increase ventilation to maintain carbon dioxide concentrations. Other theories are that the rise in body temperature may play a role, or that collateral branches of neurogenic impulses from the motor cortex to active muscles and joints may stimulate the brain stem and respiratory centre leading to hyperpnoea. Overall, a number of factors have been suggested for the increase in ventilation, which occurs with exercise. The respiratory rate might remain elevated after heavy exercise for up to $1-2 \mathrm{~h}$.

\section{Cardiovascular system}

The cardiopulmonary adaptations made to dynamic and static exercise show the amazing ability of the human body to alter physiological processes in order to meet metabolic demands. A remarkable partnership that allows individuals to maximize their abilities and obtain goals exists between the cardiovascular and pulmonary systems. The adaptations of the cardiopulmonary system depend heavily on the intensity, duration, frequency, and type of exercise being performed. Although most of this article examined dynamic and static exercise separately, the majority of individuals train using a combination of these two modes. The overall adaptations will vary with the chosen degree of each exercise mode. An appropriate exercise program allows for improvements in the cardiopulmonary system that help develop and maintain fitness levels.

Substrate and oxygen requirements of working skeletal muscles are dramatically elevated above resting requirements. Resting blood flow to muscle is usually 2-4 $\mathrm{ml} \cdot 100 \mathrm{~g} \mathrm{muscle}^{-1} \mathrm{~min}^{-1}$, but might increase to nearly 100 $\mathrm{ml} \cdot 100 \mathrm{~g}$ muscle $\mathrm{min}^{-1}$ during maximal exercise. This occurs in part because of vasodilatory metabolites such as AMP, adenosine, $\mathrm{H}^{+}, \mathrm{K}^{+}$and PO3-4P043- acting on precapillary sphincters, which override the vasoconstrictor effects of norepinephrine. In addition, decreased $\mathrm{pH}$ and increased temperature shift the oxygen dissociation curve for haemoglobin to the right in exercising muscle. This assists in unloading more oxygen from the blood into the muscle. During muscular contraction, blood flow is restricted 
briefly but overall it is enhanced by the pumping action of the muscle.

Whilst muscle and coronary blood flow increase, cerebral blood flow is maintained constant and splanchnic flow diminishes. However, essential organs such as the bowel and kidneys must be protected with some blood flow maintained. An additional demand on blood flow during exercise is the requirement to increase skin blood flow in order to enable heat dissipation.

\section{Circulatory changes}

The increase in blood flow to muscles requires an increase in the cardiac output, which is in direct proportion to the increase in oxygen consumption. The cardiac output is increased by both a rise in the heart rate and the stroke volume attributable to a more complete emptying of the heart by a forcible systolic contraction. These chronotropic and inotropic effects on the heart are brought about by stimulation from the noradrenergic sympathetic nervous system. The increase in heart rate is also mediated by vagal inhibition and is sustained by autonomic sympathetic responses and carbon dioxide acting on the medulla.

The efficacy of systolic contraction is particularly important in trained athletes who can achieve significant increases in cardiac output as a consequence of hypertrophy of cardiac muscle.

Comparison of cardiac function between athletes and nonathletes Heart rate and stroke volume increase to about $90 \%$ of their maximum values during strenuous exercise and cardiovascular function is the limiting factor for oxygen delivery to the tissues. Oxygen utilization by the body can never be more than the rate at which the cardiovascular system can transport oxygen to the tissues. There is only a moderate increase in blood pressure secondary to the rise in cardiac output. This is caused by stretching of the walls of the arterioles and vasodilatation, which in combination reduce overall peripheral vascular resistance. There is a large increase in venous return as a consequence of muscular contraction, blood diversion from the viscera and vasoconstriction.

\section{Maximum oxygen consumption}

As work rate is increased, oxygen uptake increases linearly. However, there is an upper limit to oxygen uptake and, therefore, above a certain work rate oxygen consumption reaches a plateau. This is termed the maximal oxygen uptake $\left(V^{*} \backslash\right.$ textsc $\{\backslash$ mathrm $\{0\}\} 2 \operatorname{maxV} \backslash$ textsc $\{\backslash$ mathrm $\{0\}\} 2$ max.

\section{The pulmonary system}

Pulmonary limitationsto V2max are evident in some situations, such as when exercising at high altitudes and in individuals with asthma or other types of chronic obstructive pulmonary disease. However, in most individuals exercising at sea level the lungs perform their role of saturating arterial blood with oxygen extremely effectively as described previously.

\section{Skeletal muscle limitations}

The factors listed above can be considered as 'central' factors in the same way that potential limitations in the skeletal muscle are considered "peripheral' factors limiting V2max. Peripheral factors include properties of skeletal muscle such as levels of mitochondrial enzymes and capillary density. As mitochondria are the sites of oxygen consumption (in the final stage of the ETC), doubling the number of mitochondria should double oxygen uptake in the muscle. Capillary density is known to increase with endurance training, with the effect of increasing transit time of blood through the muscle, and improving oxygen extraction from the muscle. It has been suggested that there is a relationship between capillary densityand V`textsc $\{\backslash$ mathrm $\{0\}\} 2$ maxV $\backslash$ textsc $\{\backslash$ mathrm \{ o\}\}2max.

In summary, a reduction in any of the factors involved in the delivery and utilization of oxygen will decrease. However, in healthy individuals carrying out whole-body maximal exercise at sea level, the ability of the cardio respiratory system to deliver oxygen to the working muscles rather than the ability of the muscles to consume the oxygen is limiting.

\section{Body temperature}

The maximum efficiency for the conversion of energy nutrients into muscular work is $20-25 \%$. The remainder is released in a non-usable form as heat energy, which raises the body temperature. In order to dissipate the extra heat generated as a result of increased metabolism during exercise, blood supply to the skin must be increased. This is achieved with vasodilatation of cutaneous vessels by inhibition of the vasoconstrictor tone. Evaporation of sweat is also a major pathway for heat loss and further heat is lost in the expired air with ventilation.

The hypothalamus is responsible for thermoregulation and it is important that this process is effective. However, during exercise in hot, humid conditions evaporative heat loss through sweating might not be able to remove sufficient heat from the body. Regulation of body temperature may fail and temperatures may be high enough to cause heat stroke. This presents with symptoms of extreme weakness, exhaustion, headache, dizziness eventually leading to collapse and unconsciousnes. [18] (Åstrand P-O, Rodahl K. Textbook of Work Physiology-Physiological Bases of Exercise, 3rd Edn. McGraw-Hill Book Company, 1986)

\section{Physical Functioning in Dialysis Patients}

Physical functioning is low in patients treated with dialysis, whether measured by objective laboratory measures (exercise testing), performance-based measures, or selfreport. In patients who are able to perform symptom-limited maximal exercise testing, the values for peak oxygen uptake (V02peak) are severely reduced-averaging about $60 \%$ of age-predicted values (ranging from 17.0 to 28.6 $\mathrm{mL} / \mathrm{kg} / \mathrm{minute}$ ). The specific limitations of V02peak in patients treated with dialysis have not been identified and are potentially numerous.

Painter et al. showed significant improvement in V02peak within 8 weeks after successful transplant. This improvement occurred without exercise training or significant improvements in hematocrit, suggesting that other physiologic limitations may be present in the uremic state. Anemia associated with renal disease has always been implicated in the limited V02 peak, and in the early trials of rHu-erythropoeitin treatment several studies measured V02peak. When increasing hematocrit from 17 to $20 \%$ to 30 to $33 \%$ there is a corresponding increase in V02peak. However, the increase in V02peak per increase in hematocrit is blunted compared to that observed in normal 
healthy subjects whose hematocrit was manipulated through phlebotomy and/or reinfusion of packed red cells.

The factors limiting exercise capacity in uremic patients are many. It is documented that these patients have low cardiac output responses to exercise, primarily due to a blunted heart rate. They also may be limited by endothelial dysfunction, which may affect the ability to divert cardiac output to the working muscles during exercise. The blunted heart rate response and/or severity if the V02peak levels are between 16 and $20 \mathrm{~mL} / \mathrm{kg} /$ minute. It is possible that more than $50 \%$ of patients are physically not capable of performing a symptom-limited exercise test-having physical functioning levels similar to or less than those of patients with congestive heart failure.

In patients who may not be able to perform symptomlimited exercise testing, physical performance testing may provide an indication of levels of functioning. Physical performance testing results are low in dialysis patients with gait speed averaging 66 to $77 \%$ of age-expected values. Lower extremity function as measured by sit-to-stand testing is reported to be severely limited, averaging less than $25 \%$ of normal age-predicted values. Self-reported physical functioning is the most commonly used assessment in dialysis patients and is also severely limited. The Physical Function (PF) scale on the SF-36 Health Status Questionnaire and the Composite Physical Component (PCS) scale are both significantly below reported age norms in dialysis patients.

Levels of self-reported physical functioning in dialysis patients are lower than those reported for patients with congestive heart failure and similar to those reported for patients with chronic obstructive pulmonary disease.

Low self-reported physical functioning has been shown to predict outcomes of hospitalization and death in large populations of dialysis patients. Higher self-reported functioning scores result in significant improvement in the odds of death, and declines in physical composite scores over 1 year increase mortality risk. Objective laboratory measurement of oxygen uptake is also identified as an independent predictor of death over 3.5 years of follow-up. The predictive value of these measures of physical functioning remains even when corrected for case mix, contributing co-morbidities, and contributing factors.

\section{Physical Activity in Dialysis Patients}

Most dialysis patients are sedentary. Data from the Renal Exercise Demonstration Project showed that 59\% of 286 hemodialysis patients participated in no physical activity beyond basic activities of daily living. Data from the USRDS Dialysis.

Morbidity and Mortality Study survey indicate that $12.4 \%$ were unable to ambulate or transfer and 35\% were categorized as sedentary. Two studies using the USRDS data have reported that patients classified as sedentary show significantly greater risk of mortality over 1 year compared with nonsedentary patients with adjustment for other variables associated with survival in this group (Table 84.3).

\footnotetext{
The Assessment of Exercise Capacity: Measuring Aerobic Exercise Capacity, Muscle Volume and Strength, and Physical Functioning
}

Before starting exercise therapy, patients are evaluated to define their suitability for exercise and to tailor individualized exercise prescriptions. Subsequent monitoring is also required to assess the effectiveness of the therapy.

Evaluation methods range from self-reported questionnaires to rigorous laboratory measures.

\section{Exercise Tolerance Tests}

The gold standard test for aerobic capacity is the VO 2 max test measuring the maximal amount of oxygen that can be consumed in response to increasing levels of exercise. In deconditioned patients with limited ability to exercise, this test can be difficult to perform satisfactorily, so the VO 2 peak test is substituted. This measures the highest possible oxygen consumption in a symptom-limited test. Exercise duration, VO 2 max or VO 2 peak, minute ventilation, maximum work rate, acid-base status, blood lactate and carbon dioxide production can all be measured during an exercise test on a treadmill or cycle ergometer. Less than $50 \%$ of dialysis patients are initially able to perform such an exercise test, and those who can complete it may achieve only $57820 \%$ of age- and gender-predicted values.

\section{Functional Capacity Tests}

Tests regularly used to assess the effects of exercise programmes in CKD patients are the 6-min walk test and sitto-stand tests (STS). STS5 (the time taken to complete 5 sitto-stand-to-sit cycles) is a surrogate measure of muscle power and STS60 (number of sit-to-stand-to-sit cycles in 60 s) is a surrogate measure of muscular enduranc. Although widely used, these tests have not been fully validated in CKD patients. In contrast the North Staffordshire Royal Infirmary walk test (NSRI walk), a combination of walking, stair climbing and descending, has been validated in non-anaemic maintenance dialysis and resistance training is performed.

\section{Monitoring and Tailoring Exercise Therapy}

The rating of perceived exertion using the Borg scale is recommended for controlling the intensity of training. The rating of perceived exertion is a subjective feeling scale that allows the patient to report the perceived intensity of aerobic exercise. For CKD patients, a suitable exercise intensity is 12-16 on the Borg scale.

At moderate exercise intensity like this, significant improvements in exercise capacity occur, but may be manifested only slowly(typically after about 12 weeks) [45] .In view of local variation in treatment regimes, and variation in co-morbidities, exercise prescriptions for HD and peritoneal dialysis patients need to be tailored individually [85] . Three basic methods of exercise programmend delivery have been applied in patients with advanced CKD: (1) a supervised outpatient programme in a rehabilitation centre, (2) a home exercise rehabilitation programme, and (3) exercise during the HD session (intradialytic). [19](National rehabilitation instritute.)

In terms of ensuring the prescribed intensity and duration of exercise, the supervised outpatient programme is the most effective, but it is associated with the highest dropout rates [86] . For intradialytic exercise, it is safer to train during the first $2 \mathrm{~h}$ of the HD session because, after $2 \mathrm{~h}$ of dialysis, shifting of fluid from the microvasculature to the interstitium during exercise can cause a rapid reduction in 
relative blood volume [87] with accompanying cardiovascular de compensation which may preclude further exercise.

\section{Exercise Training in Dialysis}

Dialysis patients will increase their physical activity if given specific information and encouragement to do so. Increasing physical activity and participation in exercise training will improve physical functioning (with increases in V02peak ranging from 5 to $42 \%$ ) and will improve performance- based measures and self-reported functioning measures. The changes are most pronounced in patients who have low selfreported physical function scores on the SF-36 (PCS scale). Because the natural course of physical functioning in dialysis patients is deterioration of physical functioning over time, even maintenance of functioning is a positive outcome that can certainly be achieved by increasing physical activity. Reported benefits of exercise training in dialysis patients are outlined in Table 84.4.

Rehabilitative strategies in old people with CKD and ESRD

\begin{tabular}{|c|c|}
\hline Early stage of CKD & $\begin{array}{l}\text { physical activity including aerobic and resistance exercises } \\
\text { producing positive effects on cardio-respiratory fitness, physical } \\
\text { function and self-reported health }\end{array}$ \\
\hline & $\begin{array}{l}\text { moderate-to high-intensity strength training improves physical } \\
\text { performance, muscle mass and quality of life }\end{array}$ \\
\hline $\begin{array}{l}\text { Multiple co-morbidities and } \\
\text { mild disability }\end{array}$ & $\begin{array}{cl}\text { individualized patient-centered approach: } \\
\checkmark & \text { physical activity including aerobic exercises } \\
\checkmark & \text { resistance exercises } \\
\checkmark & \text { proprioceptive neuromuscular facilitation } \\
\checkmark & \text { posture and balance control } \\
\checkmark & \text { joints mobilization and spine flexibility exercises } \\
\checkmark & \text { endurance, muscle strength and muscular stretching } \\
\checkmark & \text { gait training }\end{array}$ \\
\hline $\begin{array}{l}\text { Frail CKD/ESRD } \\
\text { dwelling-people }\end{array}$ & $\begin{array}{l}\text { individualized patient-centered approach according functional } \\
\text { limitations and residual ability; } \\
\text { address limitations and focus on patients priority and family or care- } \\
\text { givers participation; } \\
\text { goals-oriented interventions and functional areas of interventions } \\
\text { with supervised sessions; } \\
\text { patients and family educations; } \\
\checkmark \text { physical activity including aerobic exercises } \\
\checkmark \text { proprioceptive neuromuscular facilitation } \\
\checkmark \text { posture and balance control } \\
\checkmark \text { joints mobilization } \\
\checkmark \text { muscular stretching } \\
\checkmark \text { gait training } \\
\checkmark \text { occupational therapy } \\
\checkmark \text { speech and cognitive rehabilitation }\end{array}$ \\
\hline $\begin{array}{l}\text { Frail CKD/ESRD people } \\
\text { requiring hospitalization }\end{array}$ & $\begin{array}{l}\text { comprehensive multi-dimensional strategy and goal-oriented } \\
\text { approach; } \\
\text { discussion regarding goals of care and advance care planning ought } \\
\text { to be common place for such patients; } \\
\text { specialized ward and interdisciplinary care team; } \\
\text { geriatric hemodialysis rehabilitation care }\end{array}$ \\
\hline
\end{tabular}

(Rehabilitative strategies in old people with CKD and ESRD) 


\begin{tabular}{|c|c|}
\hline Early stage of CKD & $\begin{array}{l}\text { physical activity including aerobic and resistance exercises } \\
\text { producing positive effects on cardio-respiratory fitness, physical } \\
\text { function and self-reported health }\end{array}$ \\
\hline & $\begin{array}{l}\text { moderate-to high-intensity strength training improves physical } \\
\text { performance, muscle mass and quality of life }\end{array}$ \\
\hline $\begin{array}{l}\text { Multiple co-morbidities and } \\
\text { mild disability }\end{array}$ & $\begin{aligned} & \text { individualized patient-centered approach: } \\
& \checkmark \text { physical activity including aerobic exercises } \\
& \checkmark \text { resistance exercises } \\
& \checkmark \text { proprioceptive neuromuscular facilitation } \\
& \checkmark \text { posture and balance control } \\
& \checkmark \text { joints mobilization and spine flexibility exercises } \\
& \checkmark \text { endurance, muscle strength and muscular stretching } \\
& \checkmark \text { gait training }\end{aligned}$ \\
\hline $\begin{array}{l}\text { Frail CKD/ESRD } \\
\text { dwelling-people }\end{array}$ & $\begin{array}{l}\text { individualized patient-centered approach according functional } \\
\text { limitations and residual ability; } \\
\text { address limitations and focus on patients priority and family or care- } \\
\text { givers participation; } \\
\text { goals-oriented interventions and functional areas of interventions } \\
\text { with supervised sessions; } \\
\text { patients and family educations; } \\
\checkmark \text { physical activity including aerobic exercises } \\
\checkmark \text { proprioceptive neuromuscular facilitation } \\
\checkmark \text { posture and balance control } \\
\checkmark \text { joints mobilization } \\
\checkmark \text { muscular stretching } \\
\checkmark \text { gait training } \\
\checkmark \text { occupational therapy } \\
\checkmark \text { speech and cognitive rehabilitation }\end{array}$ \\
\hline $\begin{array}{l}\text { Frail CKD/ESRD people } \\
\text { requiring hospitalization }\end{array}$ & $\begin{array}{l}\text { comprehensive multi-dimensional strategy and goal-oriented } \\
\text { approach; } \\
\text { discussion regarding goals of care and advance care planning ought } \\
\text { to be common place for such patients; } \\
\text { specialized ward and interdisciplinary care team; } \\
\text { geriatric hemodialysis rehabilitation care }\end{array}$ \\
\hline \multicolumn{2}{|c|}{ CKD = chronic kidney disease ESRD = end stage renal disease } \\
\hline
\end{tabular}

\section{Counteracting the Impact of ESRD with Intradialytica Exercise Training}

\section{Impact of CKD}

\section{Physiologic/Clinical Impact}

- Reduced V02peak

- Elevated sub maximal exercise heart rate heart rate

- Poor control of blood pressure

- Increased use of antihypertensive Medications

- Increased adiposity

- Reduced oxidative metabolism

- Exacerbated malnutrition- inflammation complex

- Uremia and elevated solute concentrations

\section{Functional Impact}

- Reduced muscular strength

- Reduced exercise capacity

- Functional limitations in daily tasks

\section{Psychological Impact}

- Increased subjective fatigue Symptoms

- Poor perception of physical functioning

- Poor perception of general health

- Increased anxiety

- Poor mental health

- Greater experience of bodily pain

- Reduced vitality

\section{Impact of Intradialytic Exercise}

- Increased V02peak

- Reduced sub maximal exercise heart rate

- Improved blood pressure control

- Decreased requirement of antihypertensive medications

- Favorable adaptation of body composition

- Increased phosphofructokinase activity

- Reduced C-reactive proteins and increased

- Improved removal of toxins by dialysis

- Increased muscular strength

- Increased 6-min walk

- Improved gait speed and sit-to-stand testing

- Reduced subjective fatigue symptoms

- Improved perception of physical functioning

- Improved perception of general health

- Reduced anxiety

- Improved mental health

- Reduced experience of bodily pain

- Increased vitality

a. Most of these benefits are also documented in nondialysis exercise training. 


\section{Recommendations for Exercise Training for dialysis patients:-}

\section{Flexibility and Strengthening Exercises}

Most patients are able to do something more than they are currently doing. For many, the most appropriate starting point may be stretching and strengthening exercises. This will improve range of motion to assist with performance of self-care and activities of daily living. Strengthening exercises may facilitate ambulation, allowing the patient to progress to some form of cardiovascular exercise-which will provide health benefits.

Stretching exercises throughout the dialysis treatment may prevent the troublesome stiffness experienced in sitting in one place for 3 to 4 hours, and may counteract the negative and painful effects of cramping that may be experienced during the treatment. Stretching and strengthening exercises should be progressed slowly.

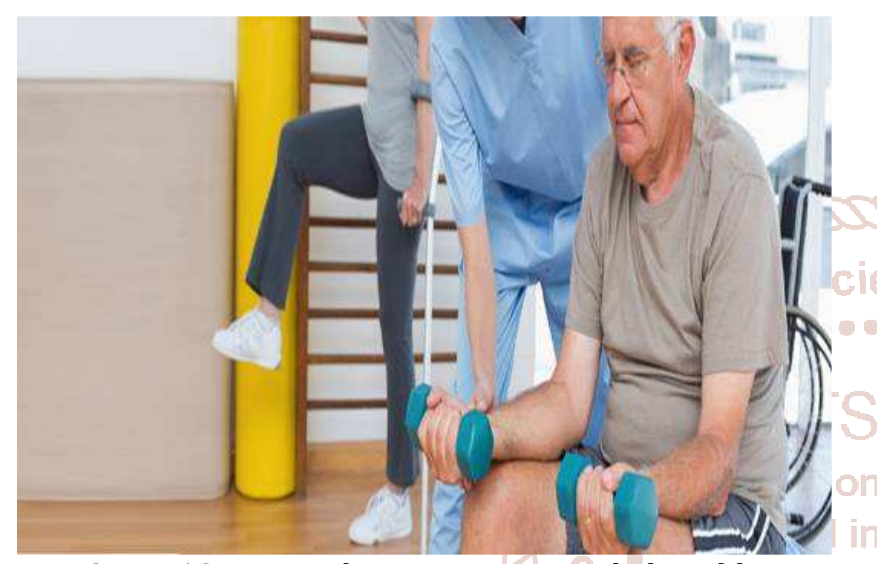

Figure 18:- strengthening exercise with doumbles

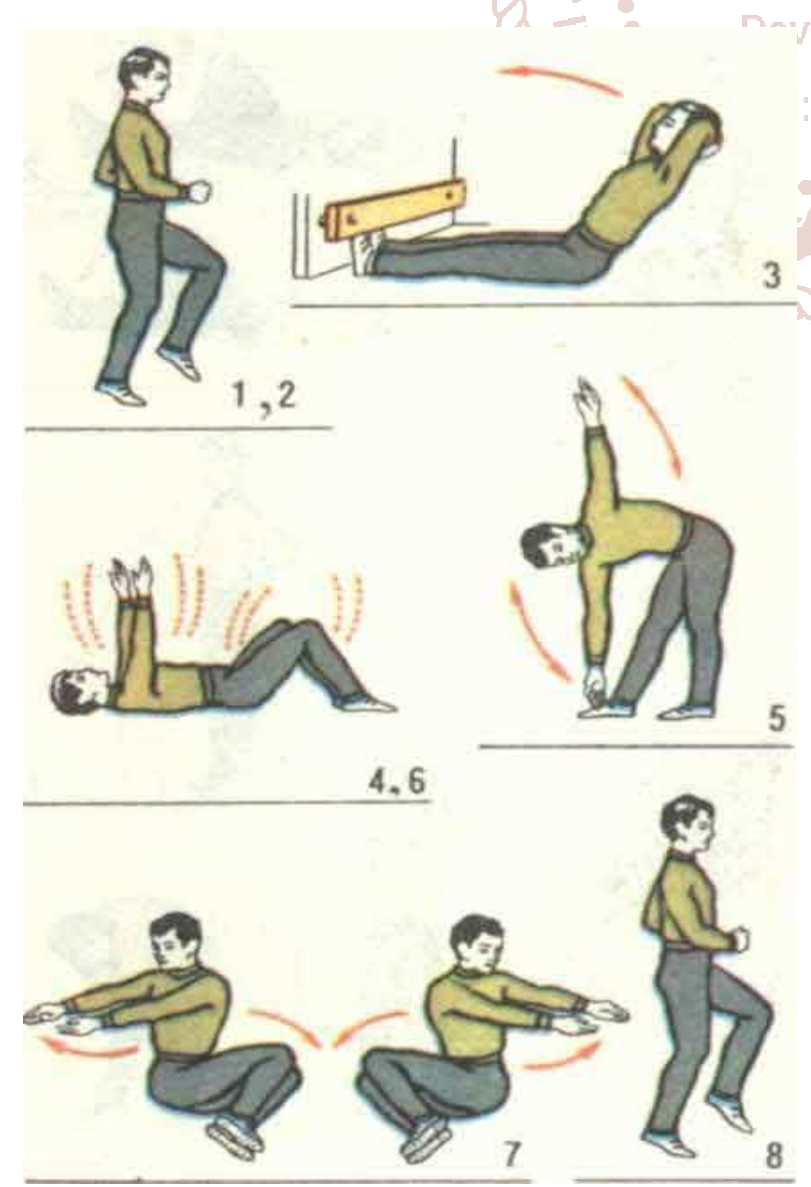

Figure 19:- active exercises for mobility.

\section{Cardiovascular Exercise Training}

Exercise that uses large muscle groups in a rhythmic manner (as in walking, cycling, rowing, and swimming) elicits health benefits in the general population and in patients with renal disease. The typical exercise prescription includes recommendations on the type, frequency, duration, intensity, and progression of exercise. The key is for patients to start exercise slowly and progress gradually.

Initial levels and progression will be highly individual, depending on starting physical condition and medical conditions. The recommendation for exercise does not have to be detailed, because for most patients just increasing their physical activity throughout the day may be beneficial. Changing activity patterns such as parking further way from the clinic or the store, or walking to the corner and back, may be helpful.

For many patients, exercise training with more structure may be recommended. Although there are no known contraindications to any type of exercise for dialysis patients, many patients experience some musculoskeletal discomfort. Thus, activities that minimize jarring on the joints may be most appropriate-specifically, walking, cycling, or swimming. Exercise should be encouraged on most days of the week. However, nondialysis days may be most convenient for exercise due to excessive fatigue experienced following the dialysis treatment-as well as the time required for the treatment.

Patients initiating cardiovascular exercise should start with a duration of exercise that is comfortably tolerated (i.e., 5 or 10 minutes). Duration should gradually increase by 2 to 5 minutes per session each week as tolerated to a goal of 30 to 45 minutes per session. Some patients may need to do intervals, meaning that the exercise is performed for 1 to 2 minutes followed by 1 to 2 minutes of rest (repeated as tolerated). Gradually the rest intervals will be decreased so that the patient works up to a continuous session of 30 to 45 minutes. Although the intensity of exercise is usually gauged by heart rate, this is not recommended for dialysis patients due to blunted chronotropic response to exercise, changing fluid status, and irregular medication regimens that may change the heart rate on a given day. Thus, with dialysis patients intensity of exercise is gauged by a subjective scale called the Rating of Perceived Exertion-which is a scale from 0 to 10 (or 6-20) with descriptors of "fairly light," "light," "somewhat hard," "hard," and "very hard". The exercise session should begin with a warm-up or activity at a low intensity of "fairly light" increased in intensity to a subjective level of "somewhat hard" or "hard." Use of this subjective scale to gauge intensity allows for the changing physiology and fatigue experienced from day to day between and after dialysis treatments. 


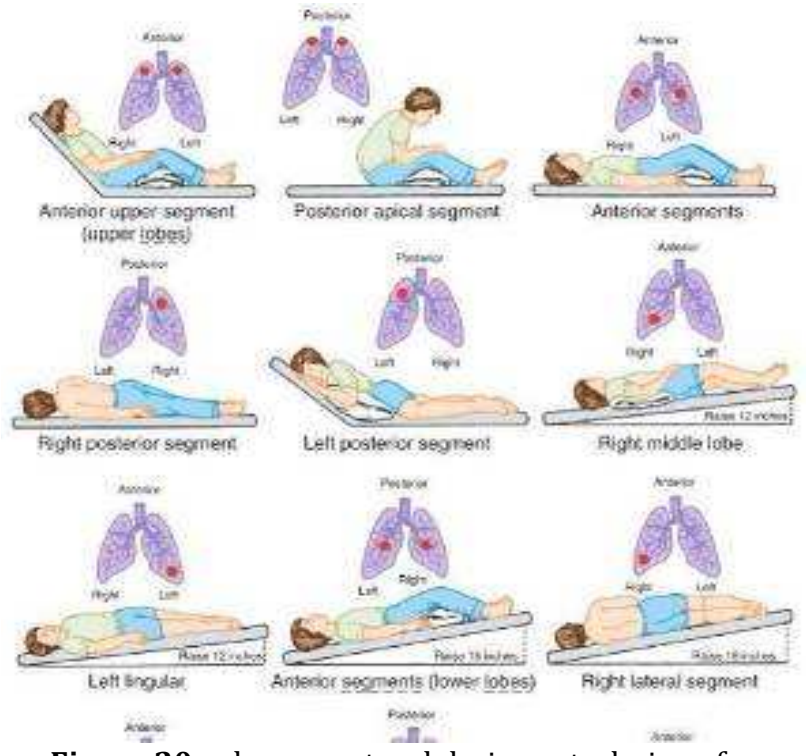

Figure 20:- shows postural drainage technique for different lobes.

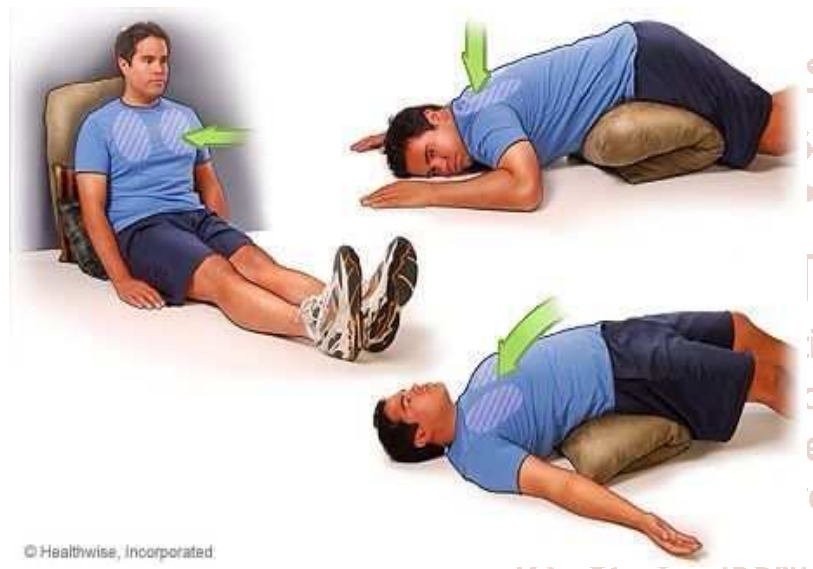

Figure 21:- relaxation position for breathlessness

\section{Delivery of Exercise Counseling:}

Nephrologists could provide most dialysis patients the opportunity to gain strength and endurance and improve activity tolerance by referring them to physical therapy or cardiac rehabilitation. More proactively, these services could be easily provided within the dialysis center. Exercise during the treatment is safe and convenient and allows for easy supervision by health care personnel. It has also been shown that intradialytic exercise improves clearance of toxins through increasing blood flow through the leg muscles, thus exposing greater tissue area to dialysis. Even if exercise is not feasible during the dialysis treatment, patients should be regularly encouraged by dialysis staff to exercise on their nondialysis days by including such encouragement and monitoring as part of the routine pre-dialysis assessment and by including it as part of the patient care plan, which is regularly reviewed and addressed by the care team.

\section{Risks of Exercise}

Exercise has two general types of risk: disease dependent and disease independent (the latter being the most common). Musculoskeletal injury and muscle soreness are the most common. In dialysis patients, muscle and joint soreness will be experienced because most patients have been sedentary for a long time. These are usually minor and transient. More serious disease-dependent risks include stress fractures and tendon avulsions, which may be possible in dialysis patients who have had uncontrolled secondary hyperparathyroidism for more than 4 years. Large powerful muscles such as the quadriceps can generate force that exceeds bone strength. Screening for osteodystrophy with questions about bone pain, fractures, and laboratory evidence of osteodystrophy should be considered. Patients with evidence of bone pain or with X-ray or laboratory evidence of osteodystrophy should participate in non weight-bearing exercise and only low-level strengthening exercises. Patients allowed to become deconditioned through inactivity experience significant muscle weakness, making them more susceptible to falls. Improved muscle strength may reduce the risk of falls and associated orthopedic injury.

In the majority of dialysis patients who have diabetes, loss of blood glucose control during or after exercise is a risk. Diabetic patients can safely exercise and are encouraged to do so by most diabetes care guidelines. To prevent hypoglycemia, careful blood glucose monitoring is required. Patients must learn their own glucose response to exercise and adjust their diet and insulin accordingly. If exercise is performed during the dialysis treatment, the blood glucose will be maintained by the dialysate glucose concentrationwhich acts as a sort of glycemic clamp.

Cardiac events such as myocardial infarction and sudden death are the most feared risks of exercise, in that cardiovascular disease is prevalent in the dialysis population. In the nondialysis population of people at high risk and/or persons suspected of having cardiac disease, the reported cardiac risks during exercise testing (i.e., pushing patients to near-maximal levels) are fewer than one death per 10,000 exercise tests, 4 or fewer non-fatal myocardial infarctions per 10,000 exercise tests, and approximately 5 hospitalizations per 10,000 exercise tests.

The risk of exercise training in cardiac patients participating in cardiac rehabilitation is most recently reported to be 1 cardiac arrest per 112,000 patient-hours of exercise, 1 death per 790,000patient-hours of exercise, and 1 myocardial infarction per300,000 patient-hours of exercise. Thus, the relative risk of exercise training in patients with known cardiac disease ( 1 arrest per 112,000) is actually lower than the risk of cardiac arrest during hemodialysis-which has been reported to be 1 per 11,570 dialysis sessions. To put the cardiac risks of exercise training into perspective, two studies have reported that 4 to $7 \%$ of 1000 nondialysis patients who presented with acute myocardial infarction acknowledged strenuous physical exertion preceding the event. Although the relative risk of myocardial infarction during or soon after unaccustomed exertion (i.e., in sedentary individuals) was 2 to 6 times higher than at other times, regular exercise participation attenuates the risk of cardiovascular event during strenuous exercise. When patients in the previously mentioned studies were stratified according to frequency of regular exercise, the relative risk of a cardiac event was clearly higher in those who were sedentary. Likewise, the relative risk of cardiac arrest during exercise ecompared to risk at other times was 56 times greater in men with low levels of habitual physical activity and only 5 times greater among men with high activity levels. Thus, it is possible that dialysis patients are actually at greater risk by not being physically active because the risk of cardiac events are so much higher in less active or sedentary individuals. 


\section{Minimizing Risks}

Several steps can be taken to minimize risks associated with exercise. All dialysis patients should be adequately dialyzed. Frequent assessments of dry weight should be made to

\section{Minimizing Risks of Exercise Minimizing overall risk:}

$>$ Provide guidelines and assess regularly

$>$ Start slowly and progress gradually

$>$ Monitor (assess) patient responses to increasing levels of activity

\section{Minimizing cardiovascular risk:}

$>$ Facilitate and encourage regular participation

$>$ Provide adequate dialysis

$>$ Manage ongoing medical concerns

$>$ Control hypertension

$>$ Respond to symptoms suggestive of cardiac disease

\section{Minimizing musculoskeletal risk:}

$>$ Ensure adequate calcium/phosphorous balance

$>$ Avoid high-impact activities

$>$ Ensure appropriate warm-up and cool-down

$>$ Ensure use of appropriate footwear

$>$ Avoid use of maximal efforts

Avoid volume overload. Co-morbidities such as metabolic bone disease, diabetes mellitus, arthritis, infection, anemia, and hypertension should be addressed and aggressively managed. Hypertension must be controlled and patients must be educated about the identification and management of signs and symptoms related to cardiovascular disease. Exercise should be individualized and started at low intensity, with initial increases in duration rather than intensity. A low impact(non-weight-bearing) type of exercise should be recommended to minimize musculoskeletal stress. Prolonged warm-up and cool-down times are important to enhance redistribution of blood flow and reduce the possibility of post exercise hypotension (especially important for those using stationary cycles during the dialysis treatment).

\section{The Need for Exercise Testing}

Although exercise testing is recommended prior to initiating exercise training for individuals at high risk for cardiovascular disease, the recommendation is dependent on the level of intensity of training expected. According to the American College of Sports Medicine, for those in the increased risk category with no symptoms who plan to initiate a program of moderate exercise (i.e., intensity of 40$60 \%$ of maximal capacity) no exercise test is recommended. To recommend pre-participation exercise testing for moderate increases in physical activity presents a significant barrier to participation in regular exercise. For dialysis patients, many barriers to initiating exercise already exist (e.g., socioeconomic, fatigue, and time factors).

Because dialysis patients have such low exercise capacity, the recommended intensity of exercise is usually very low (typically less than or equal to the intensity required for most activities of daily living) and does not put patients at a substantially higher risk than their daily living activities. Thus, it is questionable whether pre-participation exercise testing is necessary for dialysis patients. The diagnostic utility of the standard exercise test in dialysis patients may be limited for several reasons. The sensitivity of the exercise electrocardiogram (ECG) ranges from 50 to $90 \%$, andspecifi city ranges from 60 to $98 \%$.Test sensitivity is decreased by inadequate stress $(<85 \%$ of age-predicted maximal heart rate) and by drugs (or conditions) that alter the cardiac work responses to exercise. In dialysis patients the following may limit the diagnostic utility of the test: skeletal muscle limitations preventing achievement of adequate stress; longstanding hypertension with LVH and strain patternson the ECG, and electrolyte abnormalities that mask changes in S-T segments; and blunted chronotropic response to exercise(most patients achieve approximately $75 \%$ of agepredicted values) limiting cardiac stress. Thus, if the goal of exercise for the dialysis patients is to increase the frequency and duration of activity and to maintain function and prevent deterioration rather than elevate the patient to an arbitrary fitness and activity level less rigid guidelines for pre-participation exercise testing may be appropriate.

Although there are no data on adverse events experienced during exercise in dialysis patients, there have been no adverse events reported in any exercise testing or training study in dialysis patients. In our experience of more than 400 exercise tests in hemodialysis patients, there have been no cardiac events. Likewise, the author has extensive experience in exercise training hemodialysis patients in both clinical programs and research studies-including those with multiple co-morbidities. In more than 600 patients involved in cycling exercise during the dialysis treatment or participating in independent home exercise, there have been no adverse cardiac events or other untoward events. The lack of adverse events is probably reflective of the low level of exercise hemodialysis patients are able to sustain during training. Because of their extreme deconditioning, typical exercise training programs for these patients start at levels that are very low and are similar to the energy expenditure of many activities of daily living. For "low fi t" patients, this level of sustained physical activity is well tolerated. Routine training even at this level, with gradual increases in duration and sometimes in intensity, results in significant improvements in physical functioning and quality of life. For dialysis patients who are exceptionally healthy, even strenuous exercise is well tolerated. In fact, there are reports of patients participating in athletic competitions such as open water swimming, long-distance cycling, Olympic and Iron-man triathlons, and bodybuilding competitions. Thus, we feel that for patients who are adequately dialyzed with well-controlled hypertension and anemia exercise is safe and is an important part of overall medical management.

\section{Incorporation into Routine Care}

The first official statement recommending regular assessment of physical functioning and encouragement of physical activity for dialysis patients is found in the recently published Kidney Disease Outcomes Quality Initiative (NKFK/DOQI) clinical practice guidelines on management of cardiovascular disease (guideline 14).

The published guideline (guideline 14.1) states, "All dialysis patients should be counseled and regularly encouraged by nephrology and dialysis staff to increase their level of physical activity."The NKF-K/DOQI recommendations are for counseling and encouragement by nephrologists and dialysis staff in regard to identification of unique challenges to exercise in order to refer patients appropriately (i.e., to physical therapy or cardiac rehabilitation), measurement of physical functioning every 6 months using physical 
performance testing or questionnaires, identification of barriers to participation, and appropriate referral to cardiac rehabilitation or physical therapy. The goal of activity is to achieve cardiovascular exercise at a moderate intensity for 30 minutes on most, if not all, days of the week. Patients with low functioning should start at very low levels and durations and gradually progress to this level.

The NKF-K/DOQI guidelines also recommend that assessment and encouragement of physical activity participation should be a part of the routine patient care plan, which includes regular review that includes assessment of changes in activity patterns and physical functioning levels. It remains to be seen whether these NKF-K/DOQI guidelines will be implemented, and the question of the optimal method of incorporating exercise counseling into nephrology practice remains to be addressed. It is probable that the most effective way to motivate and encourage patients to increase physical activity and participate in regular exercise training is through changes in the routine care within dialysis clinics. It is feasible that pretreatment patient assessment include activity participation at home since the last treatment. Documentation of this can be tracked and reviewed as a part of the patient review. The goals for each patient's exercise can be included into the short- and long-term patient care plan, with subsequent regular review. Assessment of physical functioning can be performed by a patient care technician prior to the dialysis treatment every 6 months. Information of these tests and information on physical activity participation can provide the nephrologist valuable information of a change (deterioration) in physical functioning that may indicate a change in clinical status that might not otherwise be detected.

Whether the NKF-K/DOQI guidelines are adopted or not, it seems reasonable for nephrologists to adopt guidelines of practice for conditions common in CKD patients. Many of these practices Physical Activity and Functioning in Dialysis Patients 1219 include regular physical activity. Specifically, national guidelines for the treatment of hypertension (JNCVII), hyperlipidemia (NCEP Adult Treatment Panel III), and diabetes include regular physical activity as a fi rst-line recommendation for treatment. Likewise, for individuals at high risk for cardiovascular disease or with known disease regular physical activity is recommended as a part of a comprehensive treatment program. The U.S. Surgeon General's report on physical activity and health states that "Regular physical activity can help people with chronic, disabling conditions improve their stamina and muscle strength, improve psychological well-being and quality of life by increasing the ability to perform activities of daily life."[20] (Hand book of dialysis therapy p.g. no.1150)

\section{Conclusion:-}

Our patients study in 50 patient of CKD among hemodialysis , there were 30 males (60\%) and 20 females (40\%). Maximum number of patients belonged to age group of 1085 years. Mean age of ESRD patients was $53.3 \pm 12.8$. other causes of CKD included diabetes (35\%), GN (35\%), PTB (12\%), RPD (12\%), obstructive uropathy (5.7\%), and a etiology remained unknown in $5.8 \%$ cases. The patients were classified into 1 to 5 stages according to the NKFKDOQI Figure 4.5 shows the percent distribution of the patients in each stage of CKD. The highest number of the patients belonged to stage 5 ( 45 patients,) followed by stage
4 (2 patients), stage 3 (2 patients), and then stage $2(0$ patients,). Stage1 (1 patient).

The number of patients with CKD worldwide is rising markedly becoming a priority public health problem. Rehabilitation is effective and efficient in reducing the burden of disability and enhancing activities and participation for disabled people. Exercise training or comprehensive multi-dimensional strategy and goaloriented intervention should be provided in CKD and ESRD subjects according to clinical condition and functional impairments. Since severe CKD/ESRD disabled subjects present complex dysfunction, goals and expected outcomes of treatment should be discussed with the patient and caregiver. Structured prevention programs based on reducing the risk factors for CKD and rehabilitative strategies could reduce disability occurrence and related social cost.[21]( Rehabilitation Medicine and Neuro-Rehabilitation Unit, Scientific Institute, Hospital ‘Casa SollievodellaSofferenza', San Giovanni Rotondo (Foggia), Itley)

The benefits of regular physical activity in cardiovascular disease and diabetes are well established and although the evidence base in CKD needs to be strengthened, existing data indicates similar benefits. We therefore suggest that every stable patient with CKD, irrespective of age, gender, comorbidities or prior exercise experience, should be provided with specific written advice on how to safely and effectively increase physical activity to: (i) enhance confidence and self-efficacy in performing physical activities; (ii) attenuate deterioration of physical function and associated limitations in activities of daily living; (iii) increase physiological reserve; (iv) reduce comorbid events; and (v) enhance quality of life. The challenge is to engage and educate all stakeholders in developing a renal exerciserehabilitation service that is safe, feasible, sustainable and resourced to facilitate its incorporation into the integrated care of CKD patients across the entire disease trajectory. This will require the recommendation for routine monitoring and documentation, within electronic medical records and in national registries, of the physical function and activity levels of patients, in addition to the effects of any exercise participation. This should precipitate the consensus generation of core outcomes for the screening, assessment, monitoring, as well as evaluation of exercise training effectiveness, for people with CKD (Koufaki\&Kouidi, 2010). Renal rehabilitation services should include (clinical) exercise scientists alongside physiotherapists to support the development and evaluation of individualised, effective and sustainable physical activity and exercise plans. The role of these individuals and their activities, will be central to the managed transition of rehabilitation services towards community based pre-dialysis (stages 2-4) and posttransplantation services (akin to Phase IV cardiac rehabilitation) involving, where appropriate, self-managed physical activity plans to support sustained participation. [22](The British Association of Sport and Exercise Sciences) Now that the lifesaving/life-sustaining potential of dialysis for CKD patients has been established, it is time to address the issue of quality of life. Providing information and encouragement, increasing physical activity, and participating in regular exercise training are necessary in optimizing physical functioning-which will contribute to enhanced quality of life and may optimize outcomes. 
The amount of time dialysis patients and staff spend together invariably exerts influence. It can be an opportunity to enrich one another's lives in ways that encourage and reward healthy behavior, leading to rehabilitation as defined here. All such endeavors take time, and current constrained finances in ESRD care make this difficult because time is in short supply. Using milestones and giving guidance toward rehabilitation have to be incorporated into the obligatory contacts of treatment or there will not be time to do these things. Health promotion and rehabilitation have to be a central part of therapy, not an additional or peripheral aspect of care that receives attention only after other steps are complete. When physical, social, and sometimes vocational rehabilitation are the goals of treatment, dialysis can become holistic therapy.

Expecting people on dialysis to return to active lifestyles, whether employed or not, is not always successful. However, expecting passivity, depression, and progressive physical deterioration will usually lead to just those outcomes. The social environment in a dialysis facility is conditioned by the expectations of clinicians and their response to good results. Rehabilitation will not occur passively. The matrix of adequate use of effective technology, good nutrition, appropriate medication, and medical monitoring is fundamental to dialysis care and essential for effective rehabilitation. A conscious, organized plan to help each patient improve according to individual capability is the next step toward improved health. Working partnerships between patients and clinicians can succeed in promoting greater health, confidence, and positive attitudes. Those attributes are themselves one level of rehabilitation for many patients.

Rehabilitation, and the promotion of optimal health accompanying it, is best seen as the central goal of treatment-not as an addition to treatment. Incorporated into the framework of care in this fashion, it is effectively addressed as part of routine care as much as dialysis, medication, and diet. Once clinicians are comfortable with their knowledge of renal rehabilitation, the contact with patients includes observations, recommendations, instructions, and assessments of their health status without a great increase in contact time.

Programmed exercise before, during, or after dialysis requires some oversight, which will consume time for a staff member able to supervise exercise. Surveys for health and functional status and quality of life measurement are largely self-administered, but scoring and handling requires some staff effort. Analyzing and using these results in focusing attention and improving care takes thought and practice. Helping patients to make themselves well is achievable. The rewards of improved productive living for patients, improved satisfaction for staff, and stabilizing the patient population (which preserve facility income) make renal rehabilitation a positive experience for all concerned.

While there are still no large randomized controlled trials evaluating the survival benefits of exercise, in our opinion, the evidence reviewed above justifies the regular use of exercise programmes in patients with advanced CKD. The correlation of increased mortality with low physical activity, low muscle mass and reduced physical functioning provides a clear rationale for exercise in this group of patients. It should be emphasized that the most frail and incapacitated patients are probably those most in need of physical rehabilitation as a part of their clinical care. Furthermore, there are likely to be significant gains for healthcare systems, by reducing the collateral costs of CKD patients who, because of deconditioning, require assisted transport and interventions by nurses, physiotherapists and other staff to improve mobility, and have an increased risk of falls and associated complications. Such cost savings may in the long term overcome the financial limitations which tend to impede the introduction of exercise as a routine measure in renal units.

There is clearly a pressing need for high-quality grade A evidence from randomized controlled trials in this field to settle the question of whether physical inactivity causes increased mortality in these patients or is just an indicator of poor general condition that increases mortality risk. The importance of this question can only increase in view of the rising incidence of CKD.[23] (hand book of dialysis therapy)

\section{Abbreviation :-}

ADL Activity daily life

BI Barthel index

CKD Chronic kidney disease

ESRD End stage renal disease

GCS Glass coma scale

HD Hemodialysis

HRmax Heart rate maximum

LE Light exercise

MMT Manual muscle testing

MDRD Modified of diet in renal disease.

PE ientific Pleural effusion

PO Pulmonary oedema

PSC Physical functioning score

QOL Quality of life

$\mathrm{RE} \quad$ Resistance exercise

\section{Referances:-}

[1] Rehabilitation Medicine and Neuro-Rehabilitation Unit, Scientific Institute, Hospital 'Casa SollievodellaSofferenza', San Giovanni Rotondo (Foggia), Italy. .

[2] clinical journal of the American society of nephrology

[3] hand book of dialysis therapy.

[4] (American Journal of Physical Medicine \& Rehabilitation, 2001.80: p. 13-18.) .

[5] (Bohannon, R.W., Measuring knee extensor muscle strength. American Journal of Physical Medicine \& Rehabilitation, 2001. 80: p. 13-18.)

[6] (Physical Rehabilitation, 5 $5^{\text {th }}$ edition, Susan B O’Sullivan/ Mahoney, F, and Barthel, D, PP 62-65).

[7] (hand book of dialysis)

[8] (hand book of dialysis therapy)

[9] (Dr. James C. Wasserman, Division of Nephrology and Transplantation, Maine Medical Center, 22 Bramhall Street, Portland, ME 04102-3175)

[10] (Copyright 2002 by the National Kidney Foundation, IRenal rehabilitation and improved patient outcomes in Texas dialysis facilities. Curtin RB ${ }^{1}$, Klag MJ, Bultman DC, Schatell D.) 
International Journal of Trend in Scientific Research and Development (IJTSRD) @ www.ijtsrd.com eISSN: 2456-6470

[11] (hand bookof dialysis therapy)

[12] (C) 2014 Asian Pacific Society of Nephrology).

[13] (Åstrand P-O, Rodahl K. Textbook of Work PhysiologyPhysiological Bases of Exercise, 3rd Edn. McGraw-Hill Book Company, 1986

[14] (national rehabilitation instritute.)(intradialytic).

[15] (hand book of dialysis therapy p.g. no.1150)

\section{PROFORMA}

Name

Age/ Sex

Address

ward/bed

Date

Presenting complaints

H/o present illness

Past history of

Any renal disease

Any infectious disease

Any chronic medical disease

Personal history:

Diet

Sleep

Bowel/bladder habits

Smoking/ alcoholism

Treatment history:

For DM

For Hypertension

Family history of:

DM

HTN

\section{Examinations:}

[16] ( Rehabilitation Medicine and Neuro-Rehabilitation Unit, Scientific Institute, Hospital 'Casa SollievodellaSofferenza', San Giovanni Rotondo (Foggia), Itley)

[17] (The British Association of Sport and Exercise Sciences)

[18] (hand book of dialysis therapy)

\section{General \\ Weight \\ Pulse/BP \\ Cynosis / Clubbing \\ Oedema}

\section{Systemic Examination}

Respiratory

Cardiovascular

Per abdomen

\section{Blood investigation:-}

$\mathrm{Hb}$

TLC

DLC

RFF

Electrolytes

Lipid profile

Thyroid profile

Urine examination:-

$24 \mathrm{hrs}$. Urine output

\section{Research ane}

GFR Calculation:-

MDRD formula

Physical examination:-

MMT

Tone

BI

Diagnosis 